تأثير تغذية الاعسوقه ذات السبع نقاط.Coccinella septempunctata L على مَنْ الباقلاء الاسود Aphis fabae Scopoli بعض صفاتها الحياتية

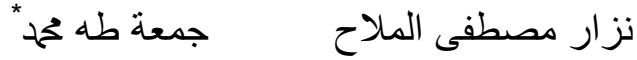

قسم وقاية النبات ـ كلية الزر اعة والغابات ـ جامعة الموصل ـ الموصل ـ العراق

E-mail: naz53ar_almlaah@yahoo.com

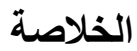

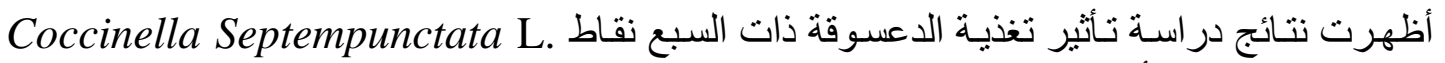

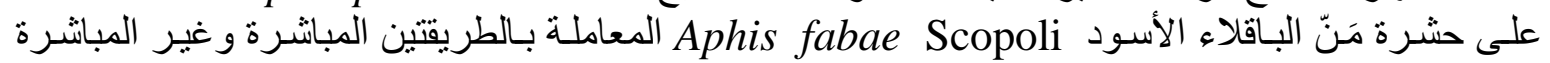

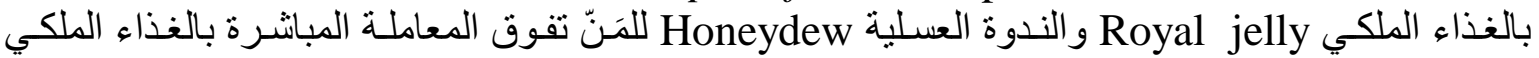

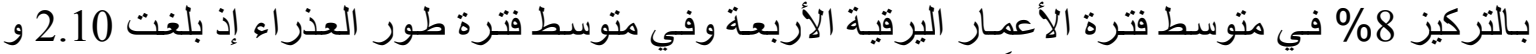

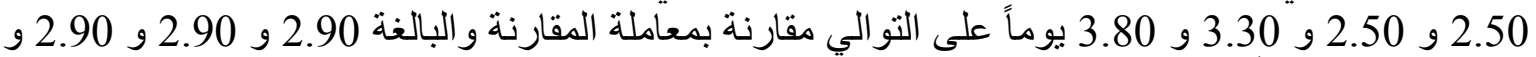

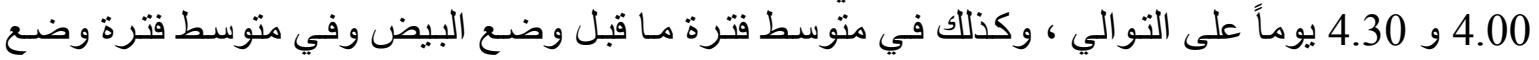

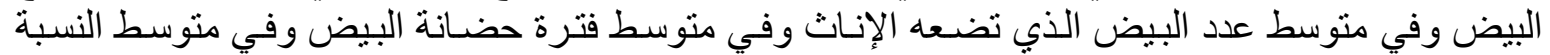

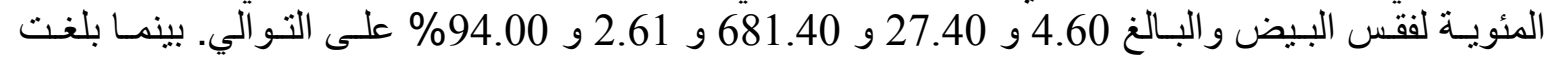

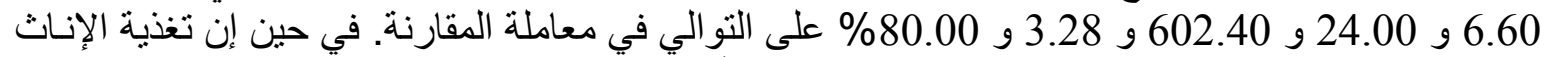

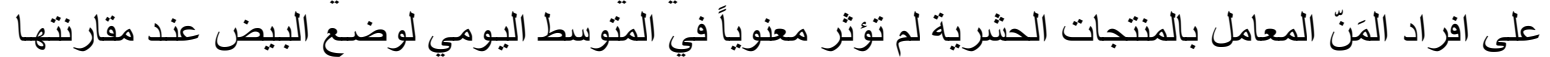

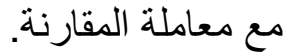
كلمّات داله:الغذاء الملكي، الندوة العسلية، الدراسـات الحياتيـه، الدعسوقة ذات السبع نقـاط ، مَنّ البـاقلاء

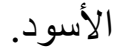

\section{تاريخ تسلم البحث: 2011/10/24 وقبوله: 2012/4/9.}

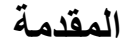

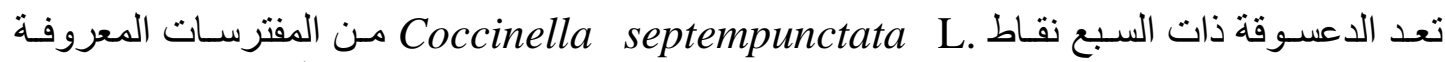

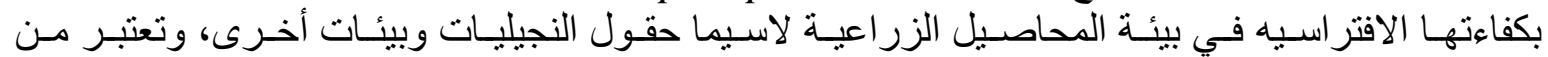

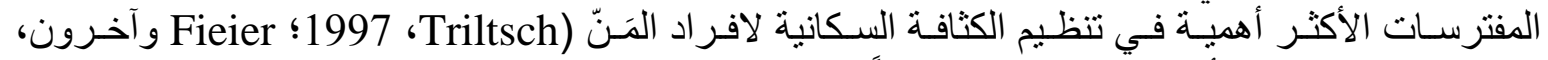

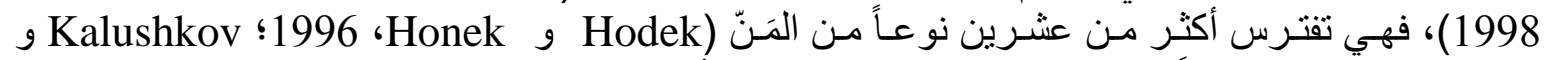
رثابلة Hodek

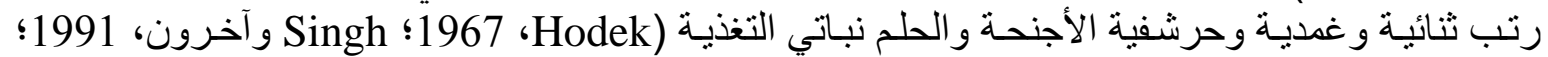
Cervez و Omkar

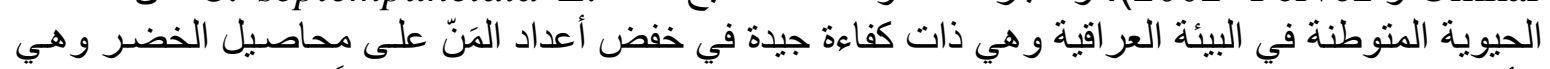

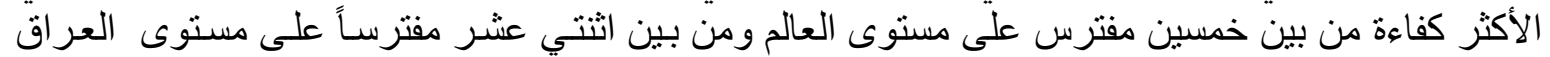
Abdul Satar)

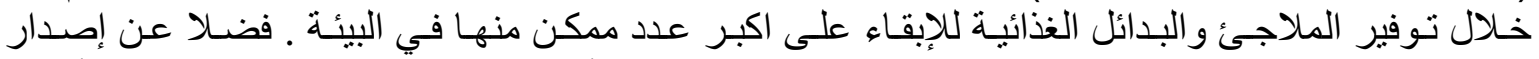

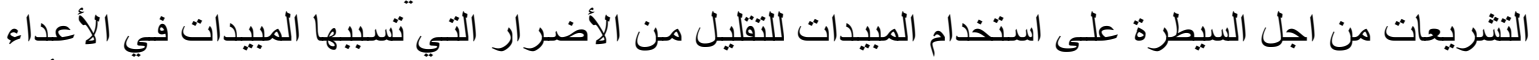

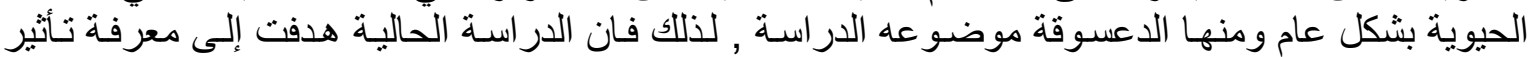

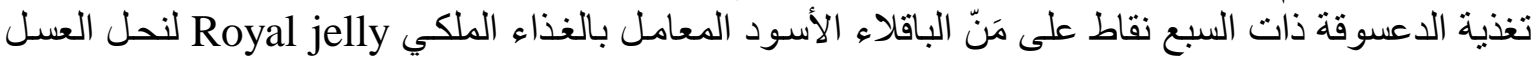

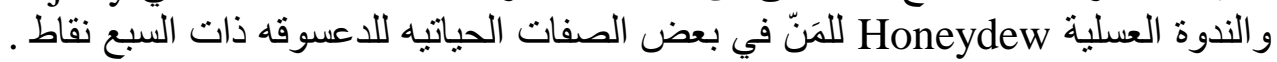

\section{مواد البحث وطرائقه}

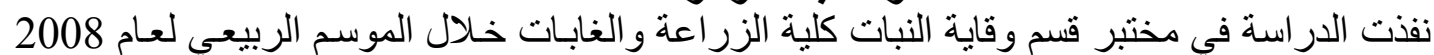

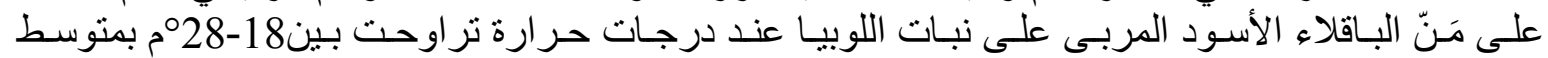

$$
\text { " مستل من أطروحة الدكتور اه للباحث الثاني }
$$


Mesopotamia. J. of Agric.

Vol.(41) No .(2) 2012
ISS:2243-4796(Online)

ISS: $1815-316 \mathrm{X}$ (Print)
مـــلجة زراعـــــــة الرافدين

المجلا (41) العدد (2) 2012

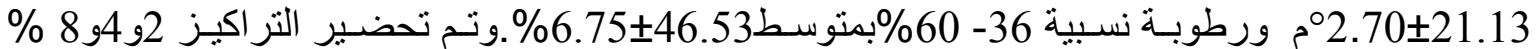

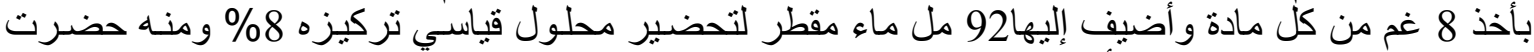

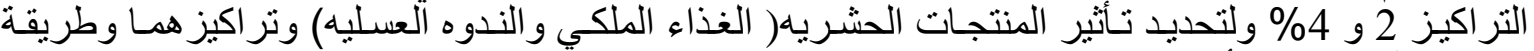

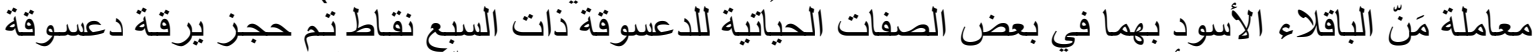

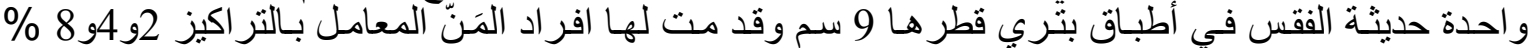

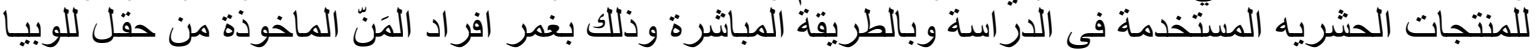

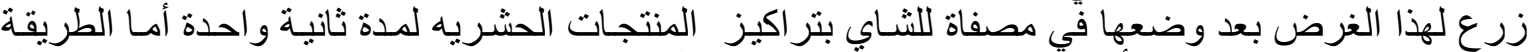

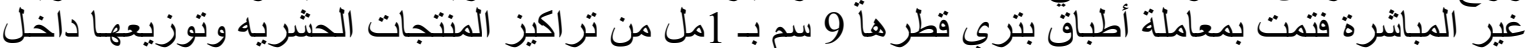

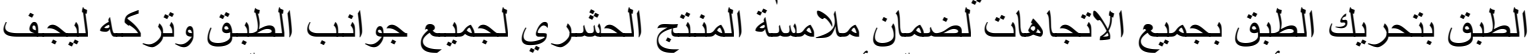

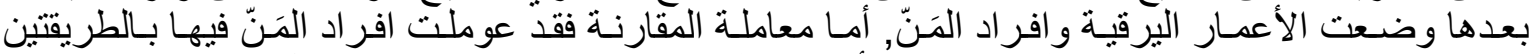

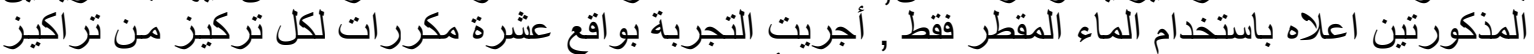

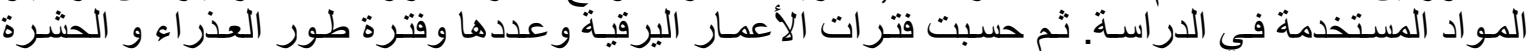

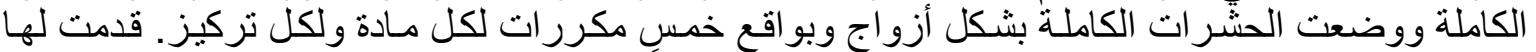

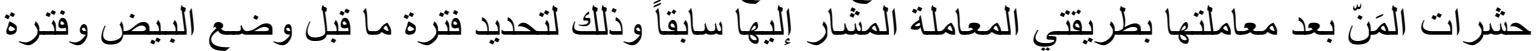

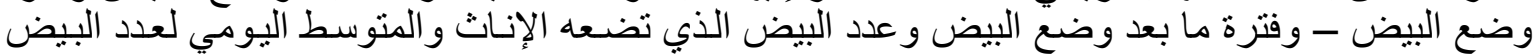

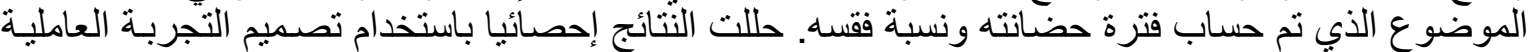

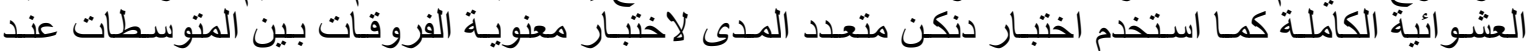

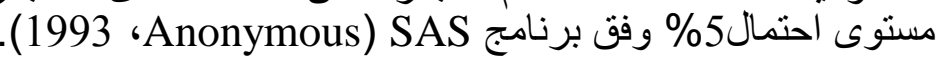

\section{النتائج والمناقشة}

1- فترة الأعمـار اليرقية: يتبين من الجدول (1) آن للغذاء الملكي و الندوة العسلية ونر اكيز همـا المستخدمة

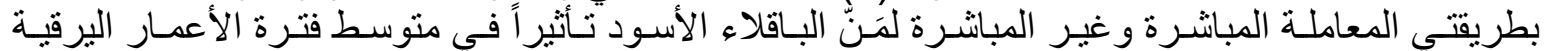

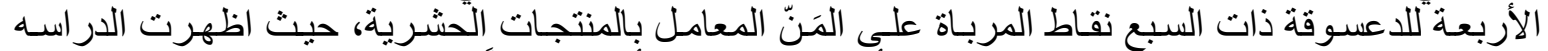

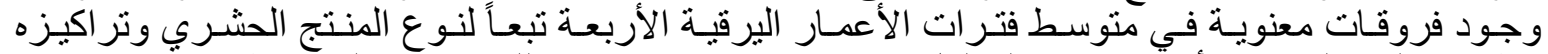

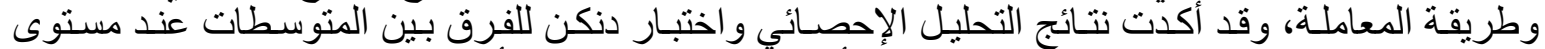

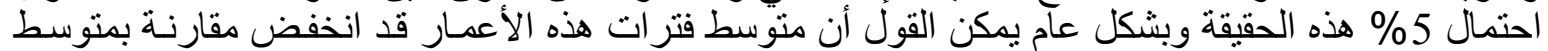

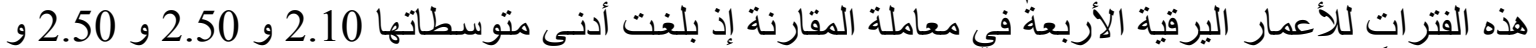

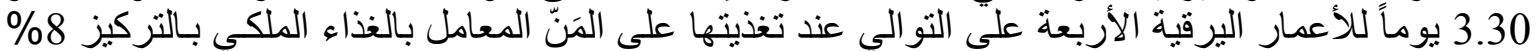

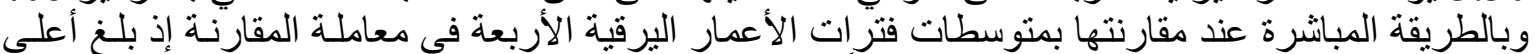

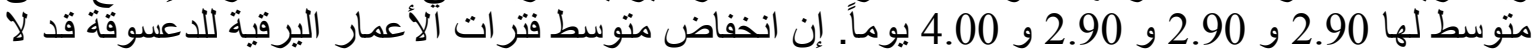

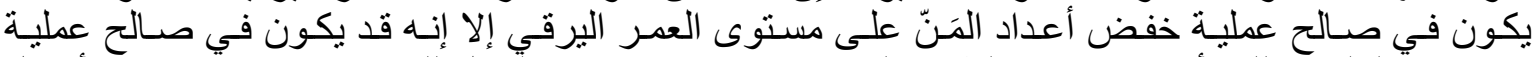

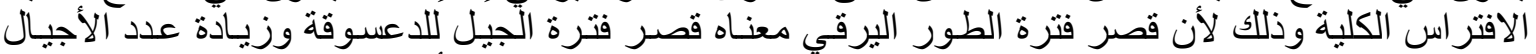

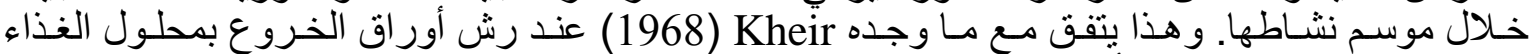

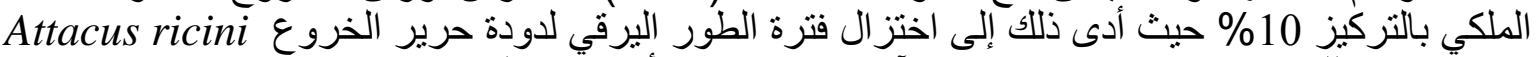

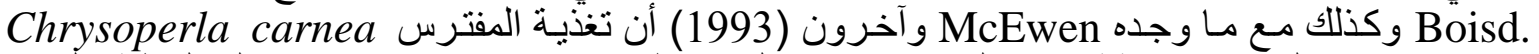
علبي بيض عثة دقيـق البحر الابيض المتوسط Ephestia kuehniella (Stephens)

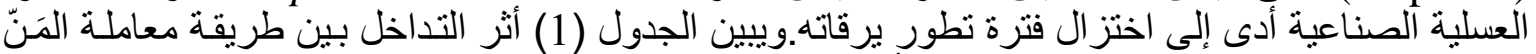

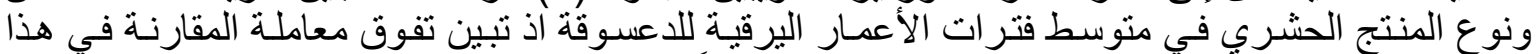

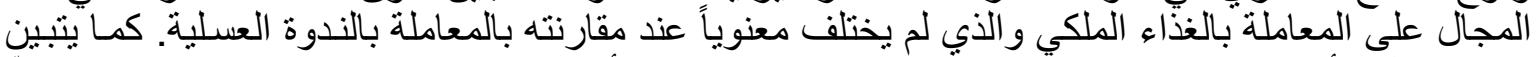

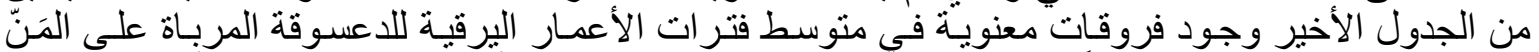

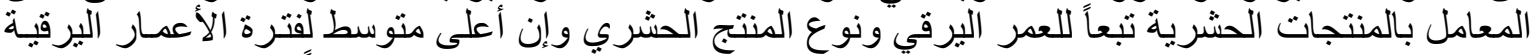

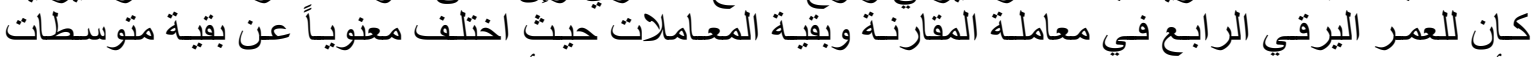

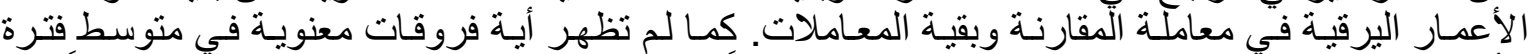

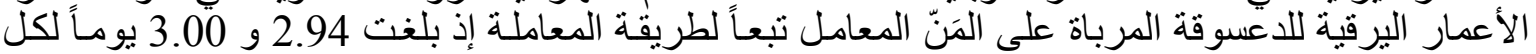

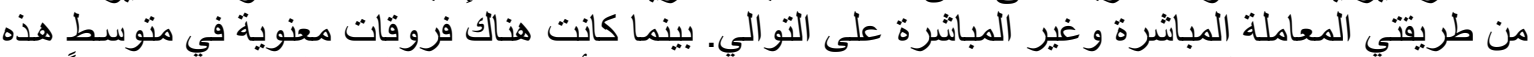

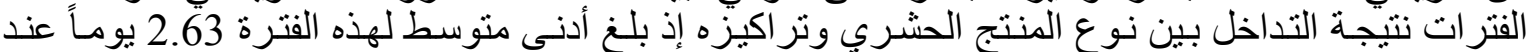

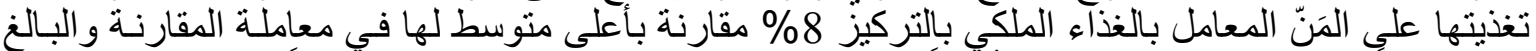

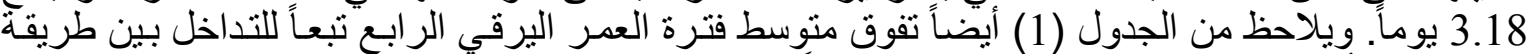

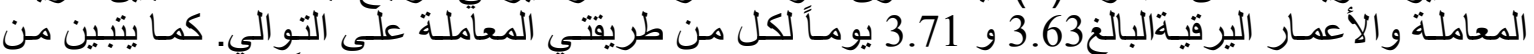

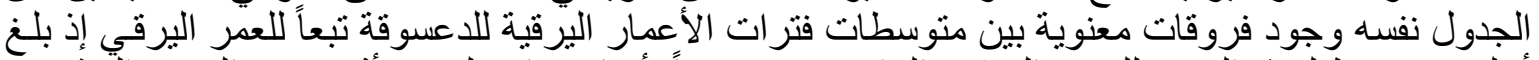

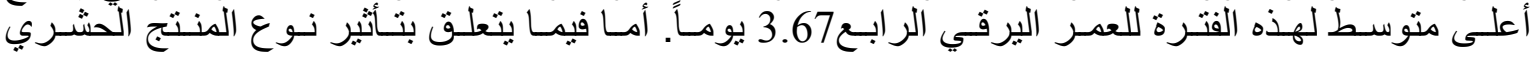


الجدول (1): تأثير تغذية الأعمار اليرقية للاعسوقة ذات السبع نقاط على مَنْ الباقلاء الأسود المعامل بطر ائق وتر اكيزمختلفه من الغذاء الملكي و الندوة العسلية في متوسط فترات عمر ها. Table.(1): Effect of feeding larvae on Aphis fabae treated by different mehod with different conc.of royal jelly and honeydew on Mean period of larval instar.

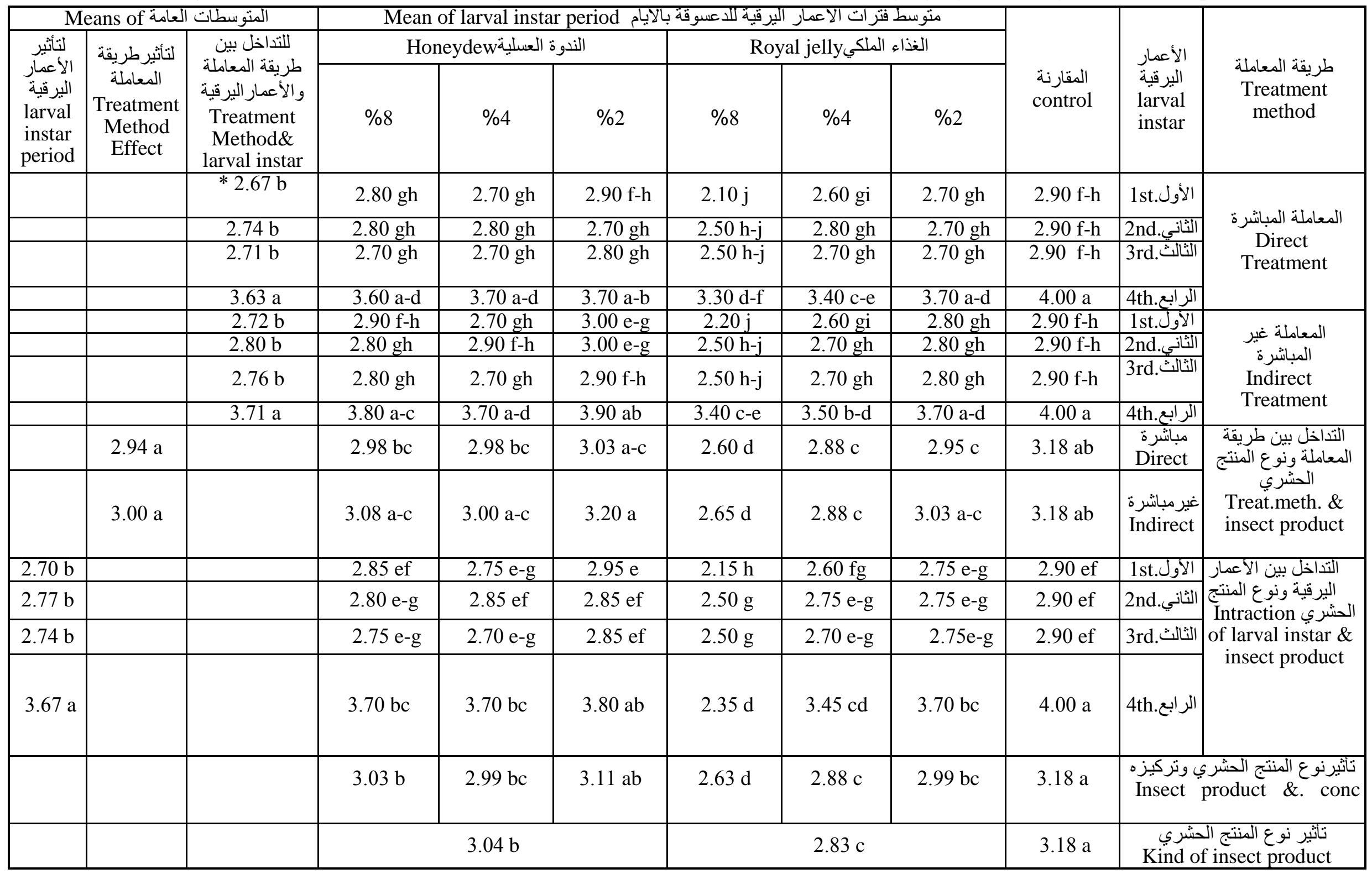

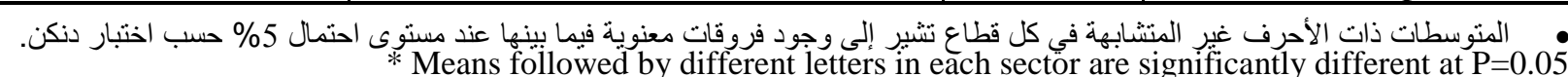




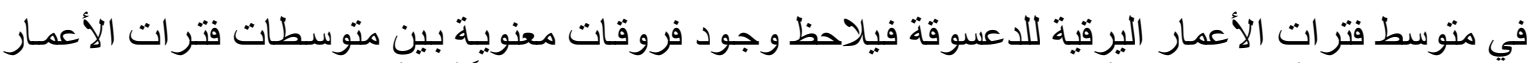

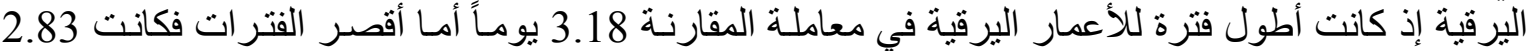

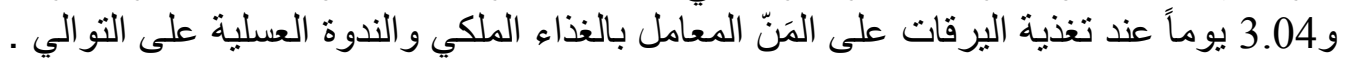

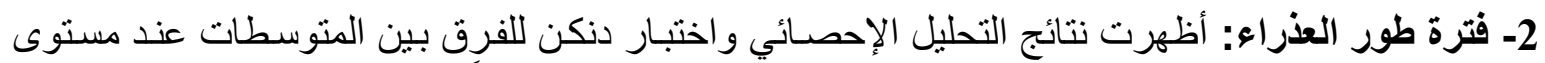

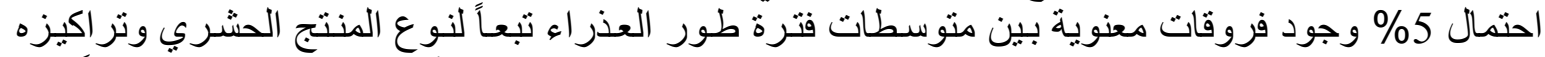

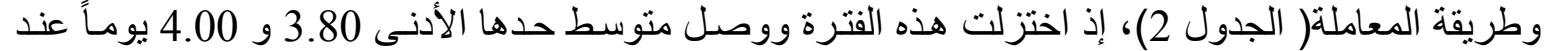

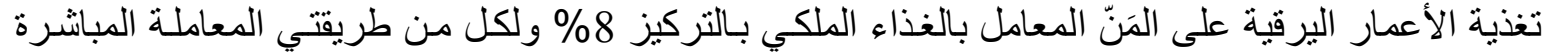

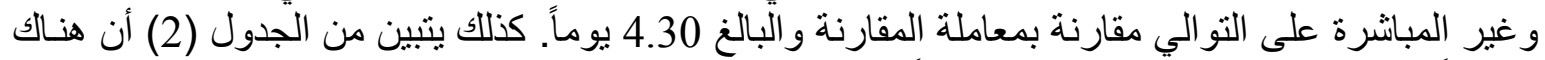

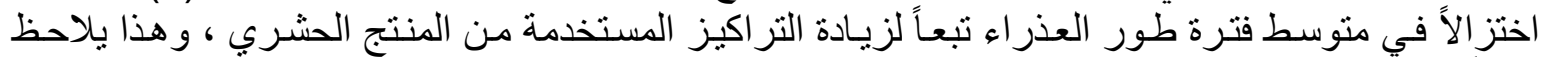

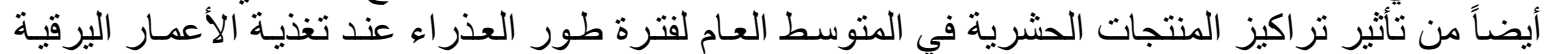

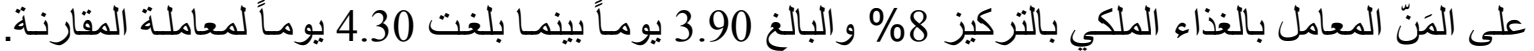

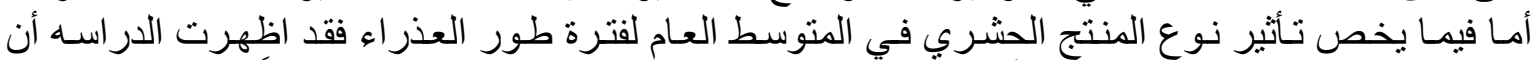

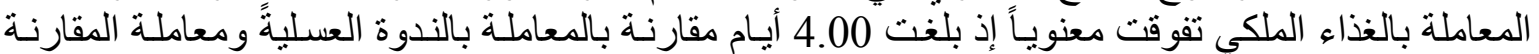

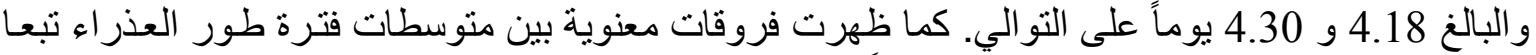

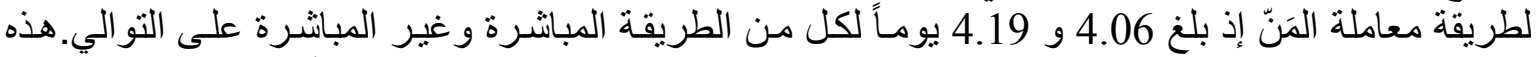

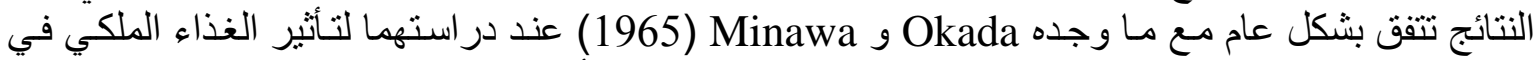

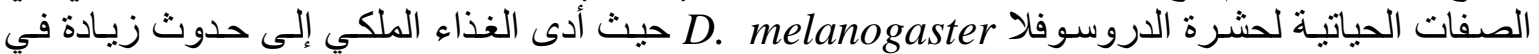

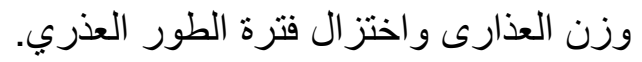

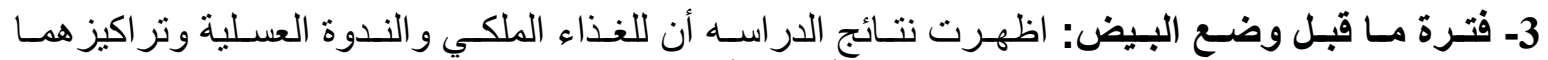

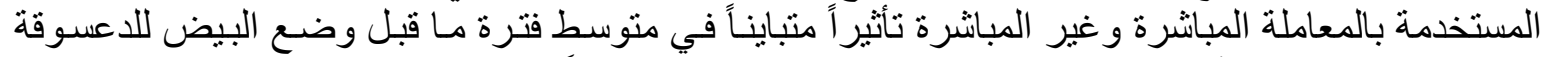

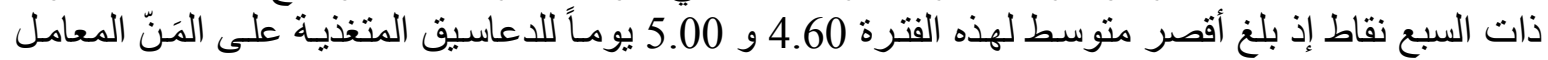

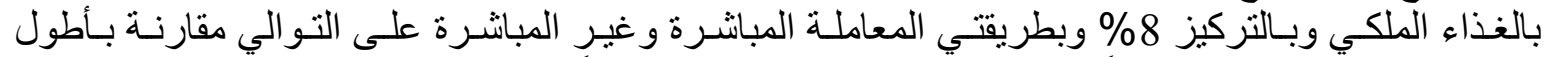

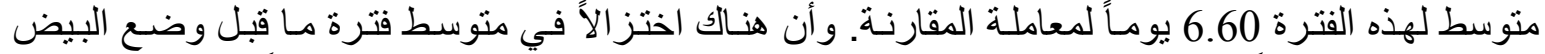

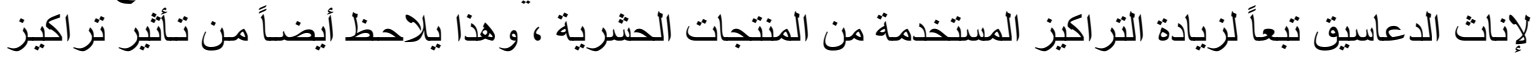

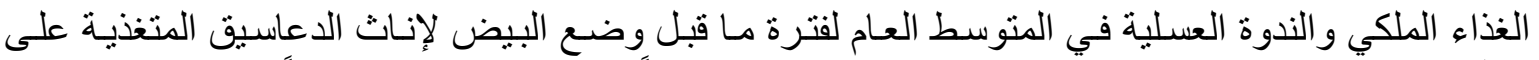

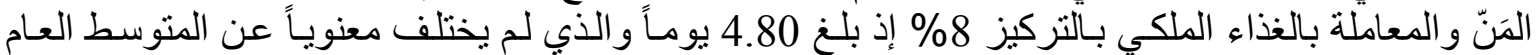

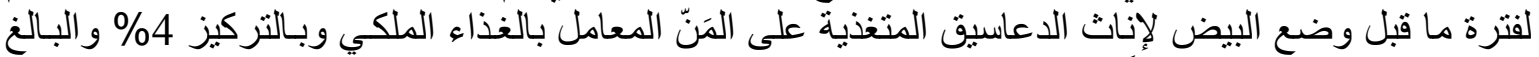

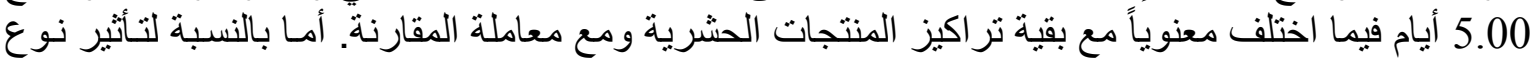

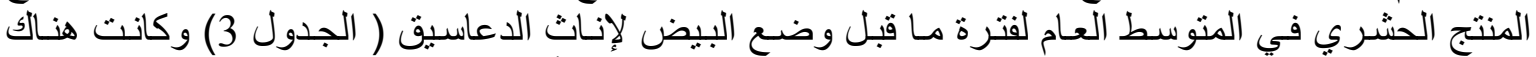

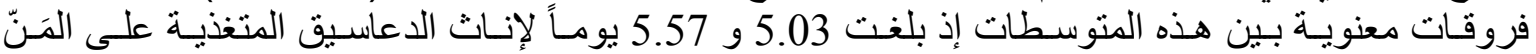

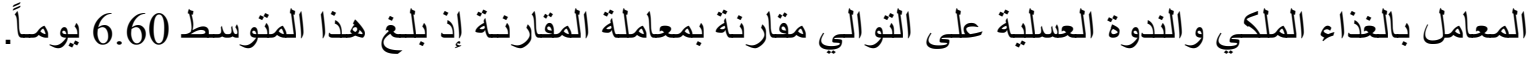

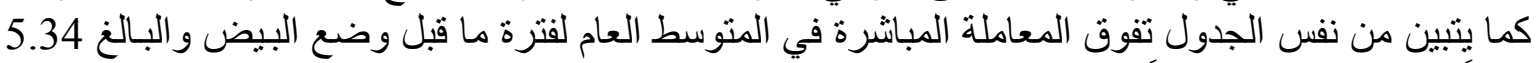

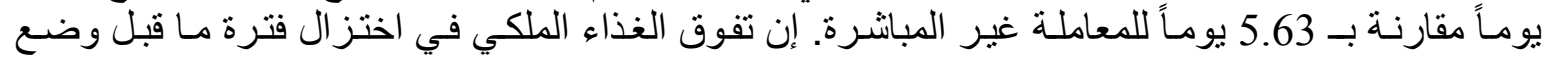

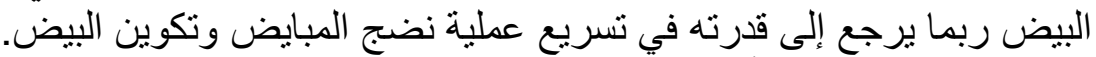

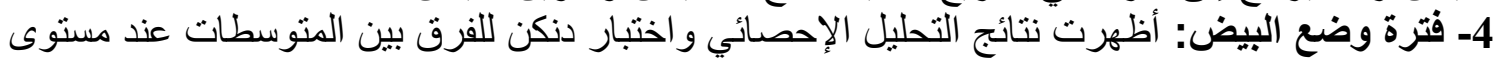

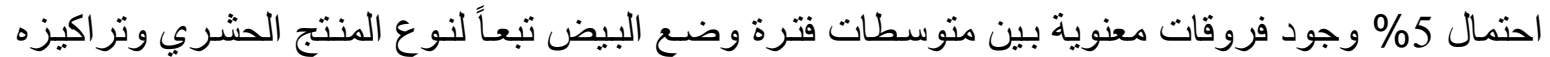

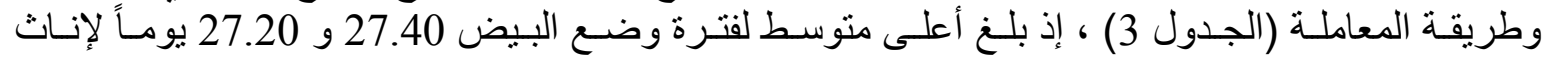

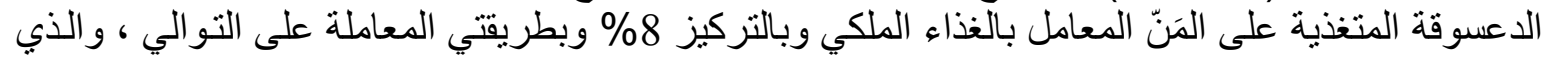

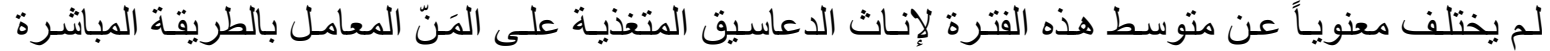

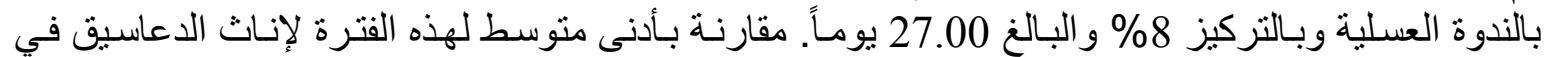

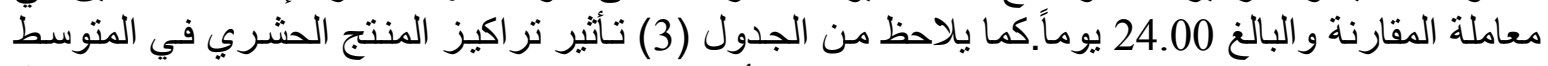

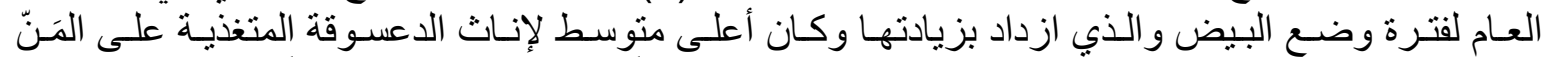

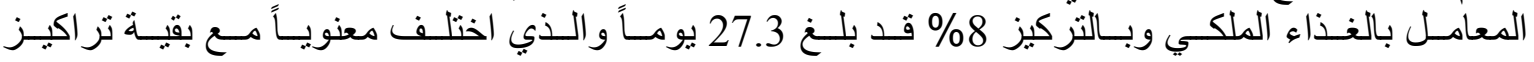


Mesopotamia J. of Agric.

Vol.(41) No.( 2)2013
ISSN: 2224-9796(Online)

ISSN 1815-316X (Print)
مجلة زر اعة الر افدين

المجلد(41) العدد (2) 2013

الجدول (2): تأثثر تغذية الدعسوقة ذات السبع نقاط على مَنّ الباقلاء الأسود المعامل بطر ائق وتر اكيزمختلفه

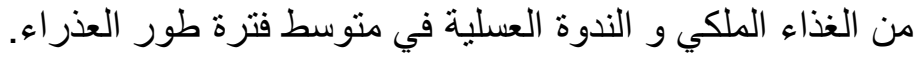

Table (2): Effect of feeding of C. septempunctata L. on Aphis fabae treated with different method \& different conc.of royal jelly and honeydew on mean pupal period.

\begin{tabular}{|c|c|c|c|c|c|c|c|}
\hline \multirow{3}{*}{ 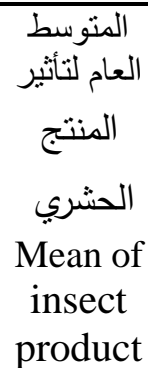 } & \multirow{3}{*}{$\begin{array}{c}\text { العام لتأثير الترنير التيز } \\
\text { Mean of } \\
\text { Conc. }\end{array}$} & \multicolumn{4}{|c|}{ 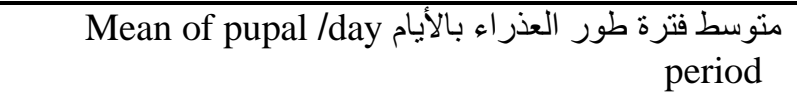 } & \multirow{3}{*}{$\begin{array}{l}\text { التز اكيز \% } \\
\text { Conc.\% }\end{array}$} & \multirow{3}{*}{$\begin{array}{c}\text { نوع المنتري } \\
\text { Kind of } \\
\text { insect } \\
\text { product }\end{array}$} \\
\hline & & \multicolumn{2}{|c|}{$\begin{array}{c}\text { المعاملة غير المباثرة } \\
\text { Indirect Treatment }\end{array}$} & \multicolumn{2}{|c|}{$\begin{array}{l}\text { المعاملة المباشرة } \\
\text { Direct Treatment }\end{array}$} & & \\
\hline & & $\begin{array}{l}\text { S.D } \pm \text { المتوسط } \\
\text { Mean } \pm \text { S.D }\end{array}$ & $\begin{aligned} \text { المدى } \\
\text { Rang }\end{aligned}$ & $\begin{array}{l}\text { S.D } \pm \text { المتوسط } \\
\text { Mean } \pm \text { S.D }\end{array}$ & $\begin{array}{r}\text { المدى } \\
\text { Rang }\end{array}$ & & \\
\hline \multirow{3}{*}{ * $4.00 \mathrm{~b}$} & $4.15 \mathrm{a}-\mathrm{c}$ & $4.20 \pm 0.42 \mathrm{ab}$ & $5-4$ & $4.10 \pm 0.32 \mathrm{a}-\mathrm{c}$ & $5-4$ & 2 & \multirow{3}{*}{$\begin{array}{c}\text { الغذاء الملكي } \\
\text { Royal } \\
\text { jelly }\end{array}$} \\
\hline & $3.95 \mathrm{bc}$ & $4.00 \pm 0.00 \mathrm{a}-\mathrm{c}$ & $4-4$ & $3.90 \pm 0.32 \mathrm{bc}$ & $4-3$ & 4 & \\
\hline & $3.90 \mathrm{c}$ & $4.00 \pm 0.00 \mathrm{a}-\mathrm{c}$ & $4-4$ & $3.80 \pm 0.42 \mathrm{c}$ & $4-3$ & 8 & \\
\hline \multirow{3}{*}{$4.18 \mathrm{a}$} & $4.25 \mathrm{a}$ & $4.30 \pm 0.48 \mathrm{a}$ & $5-4$ & $4.20 \pm 0,42 \mathrm{ab}$ & $5-4$ & 2 & \multirow{3}{*}{ | l الندوة العسلية } \\
\hline & $4.20 \mathrm{ab}$ & $4.30 \pm 0.48 \mathrm{a}$ & $5-4$ & $4.10 \pm 0.32 \mathrm{a}-\mathrm{c}$ & $5-4$ & 4 & \\
\hline & $4.10 \mathrm{a}-\mathrm{c}$ & $4.20 \pm 0.42 \mathrm{ab}$ & $5-4$ & $4.00 \pm 0.00 \mathrm{a}-\mathrm{c}$ & $4-4$ & 8 & \\
\hline \multirow[t]{2}{*}{$4.30 \mathrm{a}$} & $4.30 \mathrm{a}$ & $4.30 \pm 0.48 \mathrm{a}$ & $5-4$ & $0.48 \pm 4.30 \mathrm{a}$ & $5-4$ & \multicolumn{2}{|c|}{ المقارنةControl } \\
\hline & & \multicolumn{2}{|l|}{$4.19 \mathrm{a}$} & \multicolumn{2}{|l|}{$4.06 \mathrm{~b}$} & \multicolumn{2}{|c|}{$\begin{array}{c}\text { المتوسط العام لتأثير طريقة المعلة Mean of Treatment } \\
\text { Method }\end{array}$} \\
\hline
\end{tabular}

*المتوسطات ذات الأحرف غير المتشابهة في كل قطاع تشير إلى وجود فروقات معنويـة فيها بينها عند مستوى احتمال 5\%

Means followed by different letters in each sector are significantly different at $\mathrm{P}=0.05^{*}$

حسب اختبار دنكن.

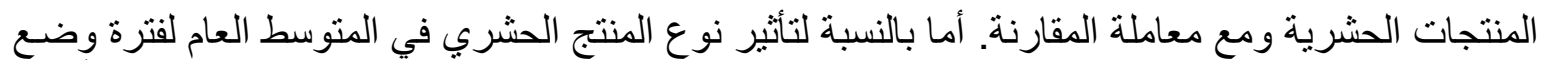

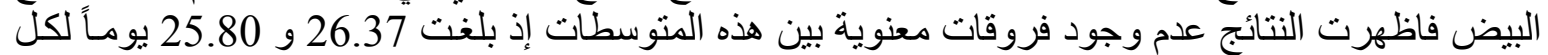

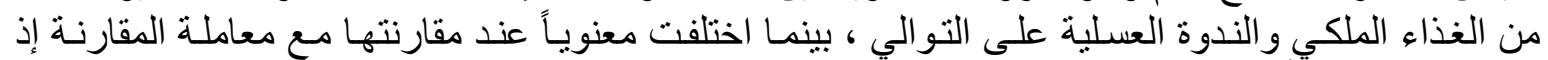

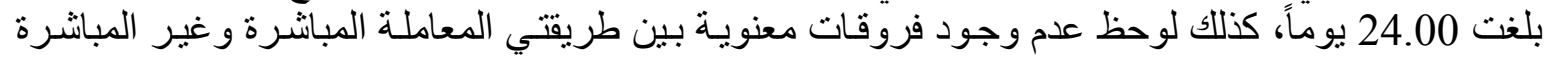

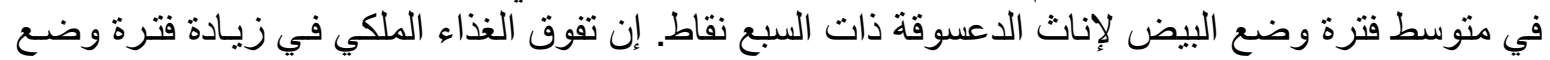

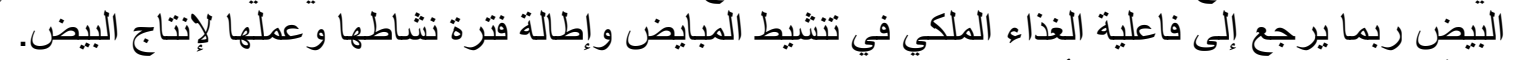

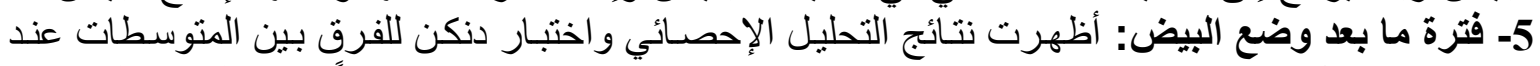

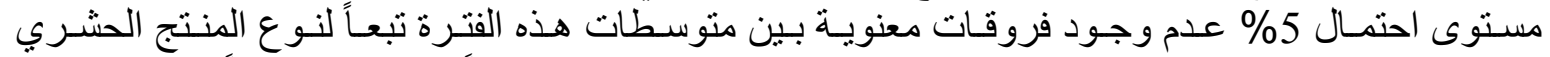

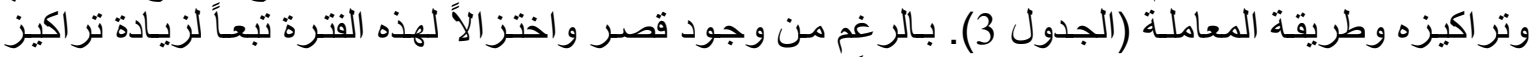

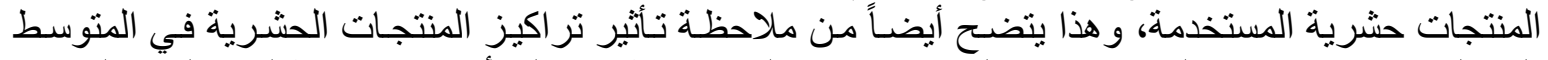

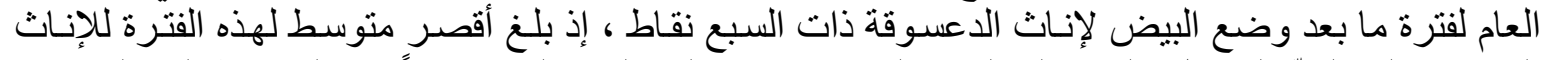

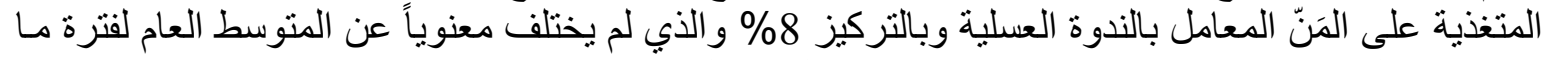

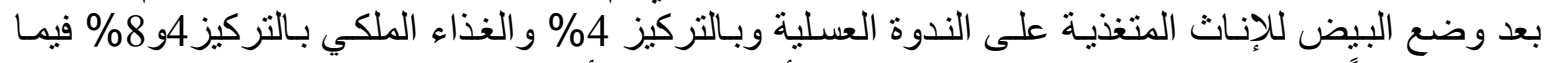

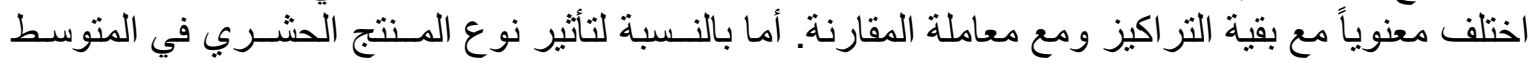


الجدول (3): تأثثير تغذيـة الدعسوقة ذات السبع نقاط على مَنّ الباقلاء الأسود المعامل بطر ائق البق

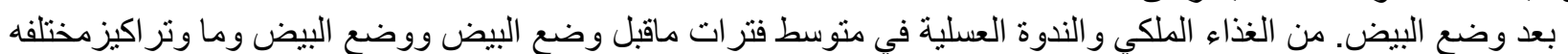

Table (3): Effect of feeding of C. septempunctata L. on Aphis fabae treated with different mehod \& different conc.of royal jelly and honeydew on mean of pre-oviposition, oviposition and postoviposition period

\begin{tabular}{|c|c|c|c|c|c|c|c|c|c|c|c|c|c|}
\hline \multirow{3}{*}{ 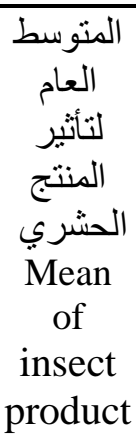 } & \multirow{3}{*}{ 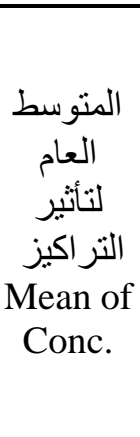 } & \multicolumn{2}{|c|}{$\begin{array}{c}\text { متوسط فترة مابعد وضع البيضام } \\
\text { postoviposition period / } \\
\text { day }\end{array}$} & \multirow{3}{*}{ 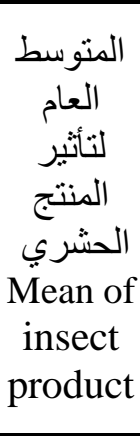 } & \multirow{3}{*}{$\begin{array}{l}\text { العام لتأثير التير اكيز } \\
\text { التئ } \\
\text { Mean of } \\
\text { Conc. }\end{array}$} & \multicolumn{2}{|c|}{$\begin{array}{l}\text { متوسط فترة وضع البيض بالياد } \\
\text { oviposition period/day }\end{array}$} & \multirow{3}{*}{ 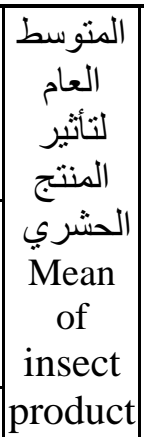 } & \multirow{3}{*}{ 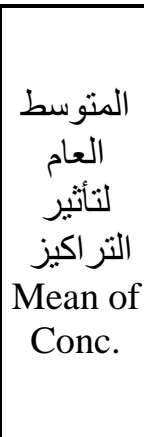 } & \multicolumn{2}{|c|}{ 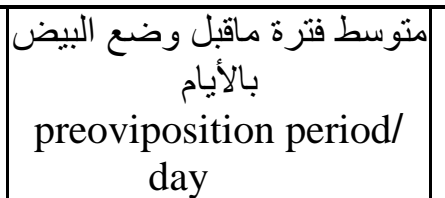 } & \multirow{3}{*}{$\begin{array}{c}\text { التر اكيز } \\
\text { Conc.\% }\end{array}$} & \multirow{3}{*}{$\begin{array}{c}\text { نوع الحنتري } \\
\text { Kind of } \\
\text { insect } \\
\text { product }\end{array}$} \\
\hline & & \begin{tabular}{|c|} 
المعاملة غياشرة \\
Indirect \\
Treatment \\
\end{tabular} & المباشرة المعاملة & & & \begin{tabular}{|c|} 
المعاملة غيرة \\
Indirect \\
Treatment \\
\end{tabular} & \begin{tabular}{|c|} 
المباملة \\
Direct \\
Treatment \\
\end{tabular} & & & \begin{tabular}{|c|} 
المعاملة غباشرة \\
Indirect \\
Treatment \\
\end{tabular} & \begin{tabular}{|c|} 
المعاملة \\
المباشرة \\
Direct \\
Treatment \\
\end{tabular} & & \\
\hline & & |المتوسطMean| & المتوسطMean & & & 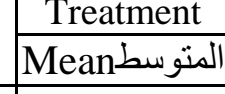 & 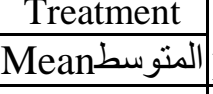 & & & |المتوسطMean & |المتوسط & & \\
\hline \multirow{3}{*}{ *5.10a } & $5.30 \mathrm{bc}$ & $5.20 \mathrm{ab}$ & $5.40 \mathrm{ab}$ & \multirow{3}{*}{$26.37 \mathrm{a}$} & $25.50 \mathrm{~cd}$ & $25.20 \mathrm{de}$ & $25.80 \mathrm{~cd}$ & \multirow{3}{*}{$5.03 \mathrm{a}$} & $5.30 \mathrm{bc}$ & $5.40 \mathrm{~b}-\mathrm{e}$ & $5.20 \mathrm{a}-\mathrm{d}$ & 2 & \multirow{3}{*}{\begin{tabular}{|c} 
الغذاء الملكي \\
Royal \\
jelly
\end{tabular}} \\
\hline & $\begin{array}{c}5.10 \\
\mathrm{a}-\mathrm{c}\end{array}$ & $5.00 \mathrm{ab}$ & $5.20 \mathrm{ab}$ & & $26.30 \mathrm{bc}$ & $26.00 \mathrm{~b}-\mathrm{d}$ & $26.60 \mathrm{a}-\mathrm{c}$ & & \begin{tabular}{|l|}
$5.00 \mathrm{ab}$ \\
\end{tabular} & $5.20 \mathrm{a}-\mathrm{d}$ & $4.80 \mathrm{ab}$ & 4 & \\
\hline & $4.90 \mathrm{ab}$ & $4.80 \mathrm{ab}$ & $5.00 \mathrm{ab}$ & & $27.30 \mathrm{a}$ & $27.20 \mathrm{ab}$ & $27.40 \mathrm{a}$ & & $4.80 \mathrm{a}$ & $5.00 \mathrm{bc}$ & $4.60 \mathrm{a}$ & 8 & \\
\hline \multirow{3}{*}{$4.97 \mathrm{a}$} & $5.30 \mathrm{bc}$ & $5.20 \mathrm{ab}$ & $5.40 \mathrm{ab}$ & \multirow{3}{*}{$25.80 \mathrm{a}$} & $25.00 \mathrm{~d}$ & $25.00 \mathrm{de}$ & $25.00 \mathrm{de}$ & \multirow{3}{*}{$5.57 \mathrm{~b}$} & $5.80 \mathrm{~d}$ & $6.00 \mathrm{ef}$ & $5.60 \mathrm{c}-\mathrm{e}$ & 2 & \multirow{3}{*}{$\mid \begin{array}{c}\text { الندوة العسلي } \\
\text { Honeydew } \\
\text { ة }\end{array}$} \\
\hline & $4.90 \mathrm{ab}$ & $5.00 \mathrm{ab}$ & $4.80 \mathrm{ab}$ & & $25.90 \mathrm{bc}$ & $25.80 \mathrm{~cd}$ & $26.00 \mathrm{~b}-\mathrm{d}$ & & $5.60 \mathrm{~cd}$ & $5.80 \mathrm{de}$ & $5.40 \mathrm{~b}-\mathrm{e}$ & 4 & \\
\hline & $4.70 \mathrm{a}$ & $4.80 \mathrm{ab}$ & $4.60 \mathrm{a}$ & & $26.50 \mathrm{~b}$ & $26.00 \mathrm{~b}-\mathrm{d}$ & $27.00 \mathrm{a}-\mathrm{c}$ & & $5.30 \mathrm{bc}$ & $5.40 \mathrm{~b}-\mathrm{e}$ & $5.20 \mathrm{a}-\mathrm{d}$ & 8 & \\
\hline \multirow[t]{2}{*}{$5.60 \mathrm{~b}$} & $5.60 \mathrm{c}$ & $5.60 \mathrm{~b}$ & $5.60 \mathrm{~b}$ & $24.00 \mathrm{~b}$ & $24.00 \mathrm{e}$ & $24.00 \mathrm{e}$ & $24.00 \mathrm{e}$ & $6.60 \mathrm{c}$ & $6.60 \mathrm{e}$ & $6.60 \mathrm{f}$ & $6.60 \mathrm{f}$ & \multicolumn{2}{|c|}{ المقارنةControl } \\
\hline & & $5.09 \mathrm{a}$ & $5.14 \mathrm{a}$ & & & $25.60 \mathrm{a}$ & $25.97 \mathrm{a}$ & & & $5.63 \mathrm{~b}$ & $5.34 \mathrm{a}$ & 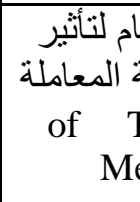 & $\begin{array}{l}\text { المتو سط الع طريقة Mean } \\
\text { Treatment } \\
\text { ethod }\end{array}$ \\
\hline
\end{tabular}

*Means followed by different letters in each sector are significantly different at $\mathrm{P}=0.05$ 
Mesopotamia. J. of Agric.

Vol.(41) No .(2) 2012
ISS:2243-4796(Online)

ISS: $1815-316 \mathrm{X}$ (Print)
مـــلة زر اعــــــــة الر افدين

المجلد (41) العدد (2) 2012

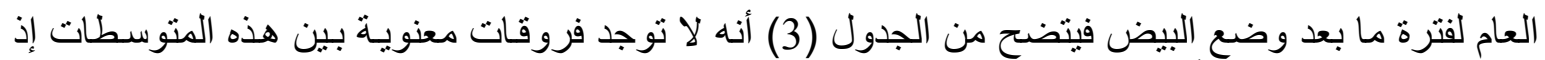

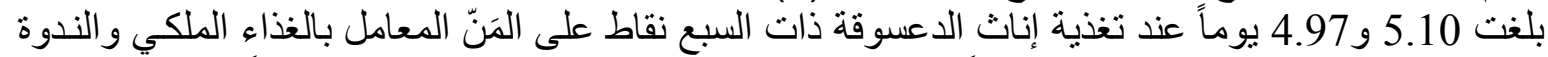

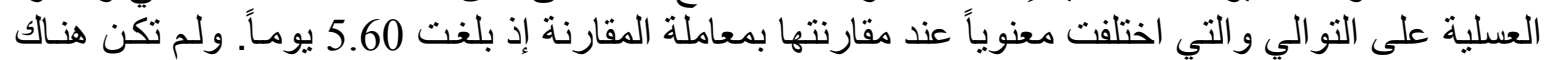

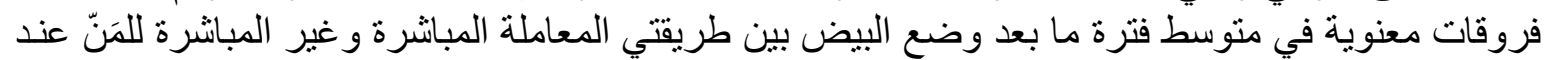

تغذية الدعاسيق عليها. 6- عدد البيض: يلاحظ من الجدول (4) أن للغذاء الملكي و الندوة العسلية وتر اكيز همـا المستخدمة بالمعاملـة

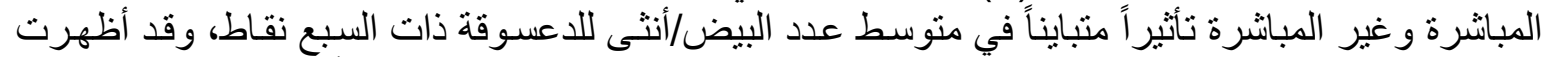

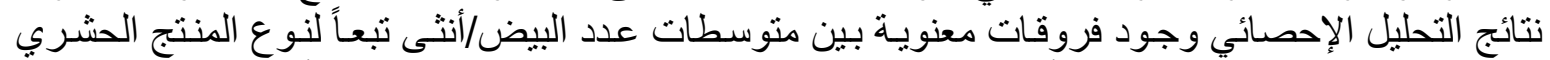
وتر اكيزه وطريقة المعأملة، إذ بلغ أعلى منوسط لعدد البيض

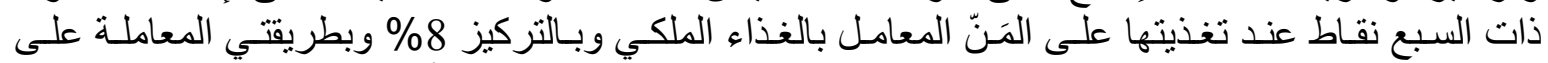

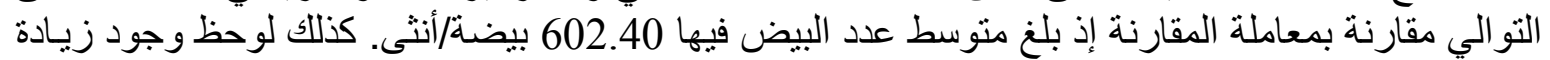

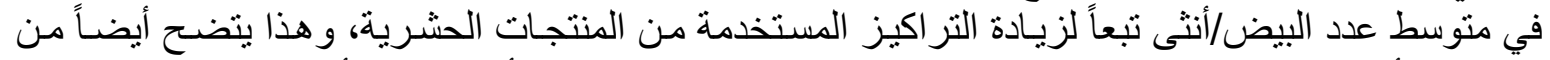

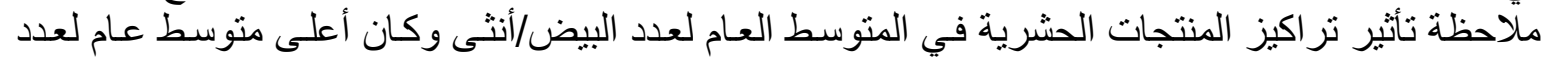

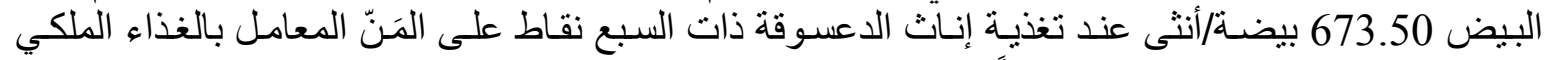

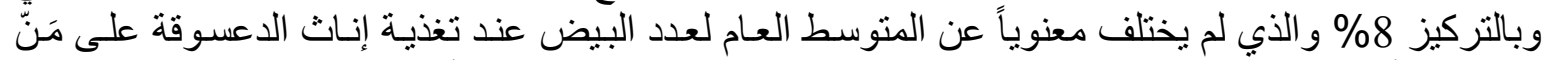

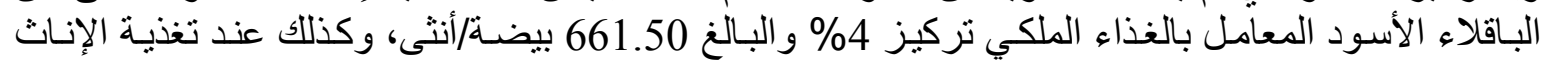

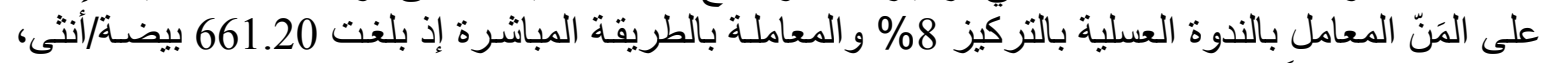

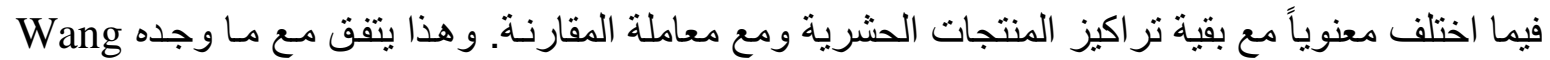

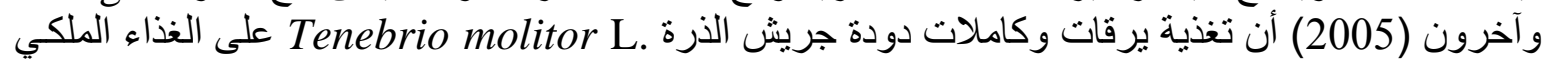

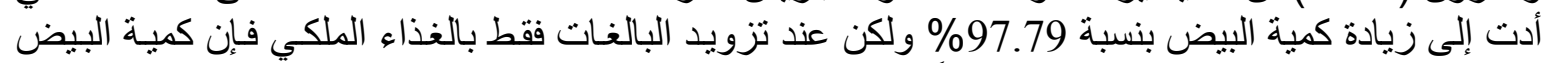

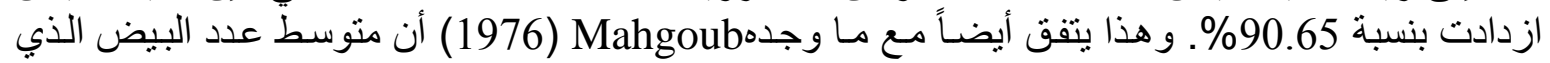
وضعته إنـاث دودة حرير الخروع .Attacus ricini Boisd قد ازدادت بزيسادة التراكيز المستخدمة من الغذاء الملكي عند معاملة أوراق الخروع بـ به إذ بلغت

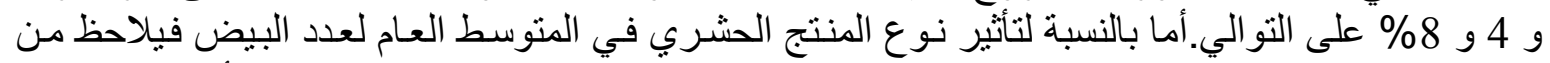

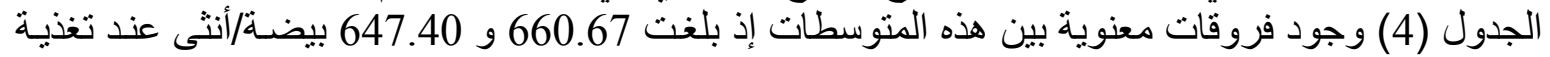

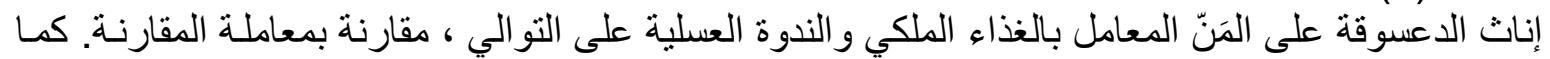

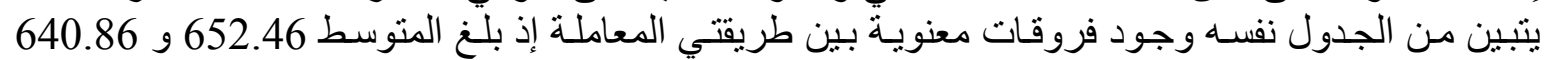

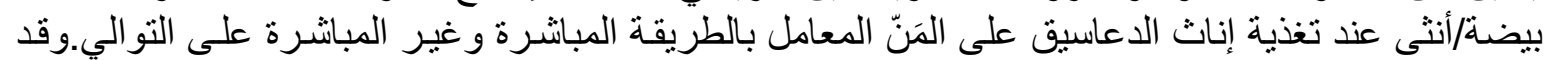

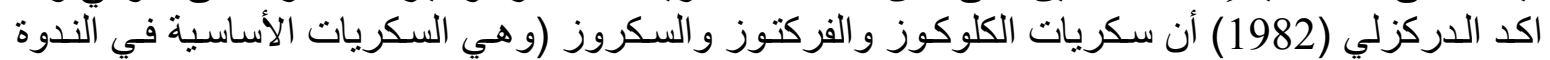

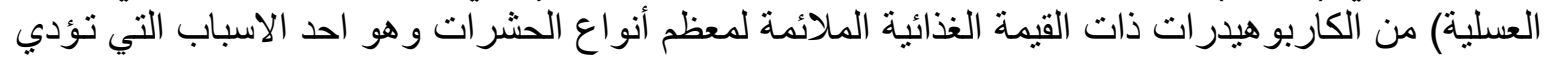

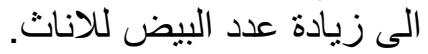

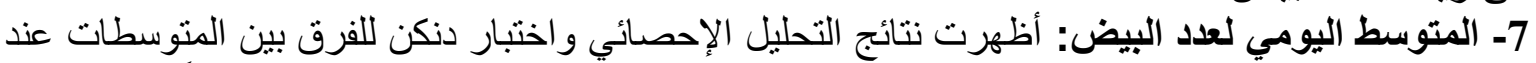

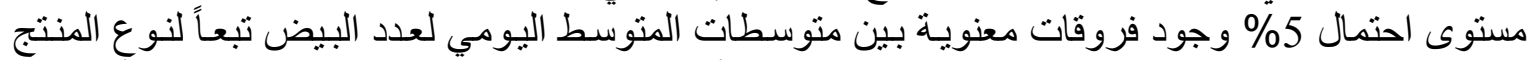

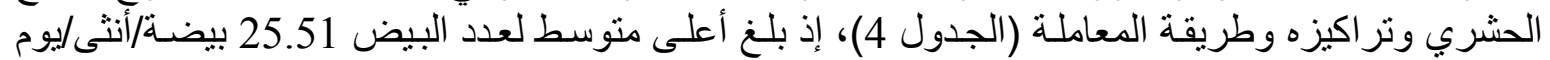

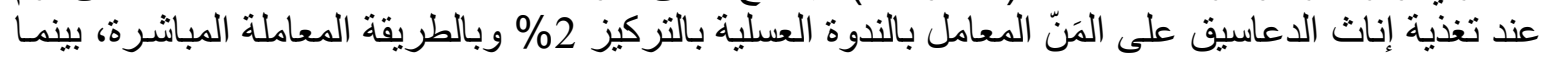

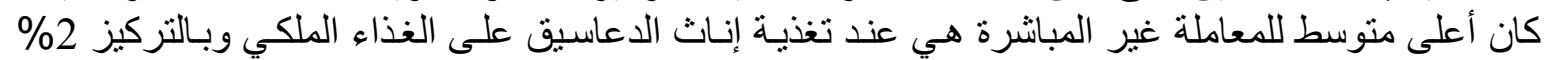

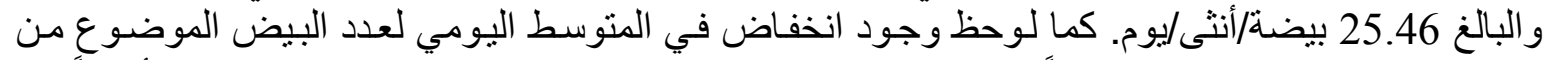

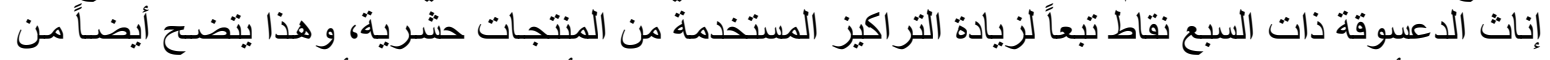

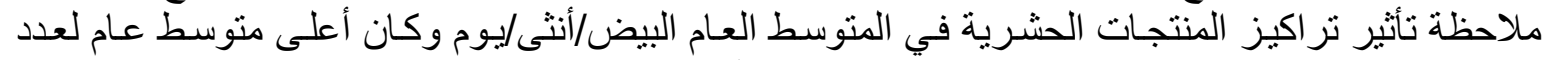

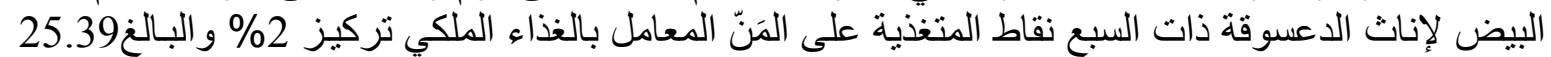

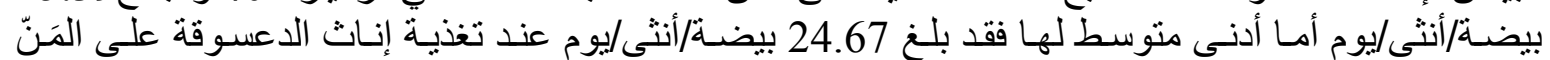

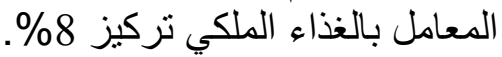

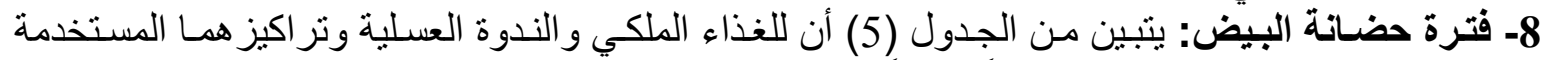

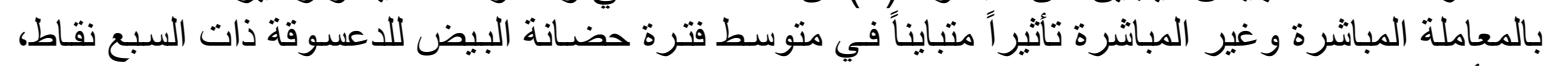

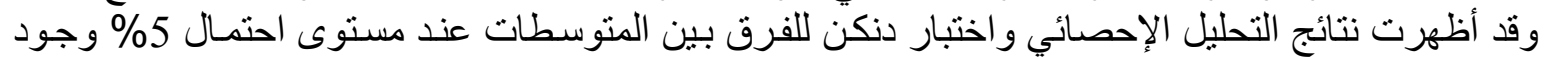

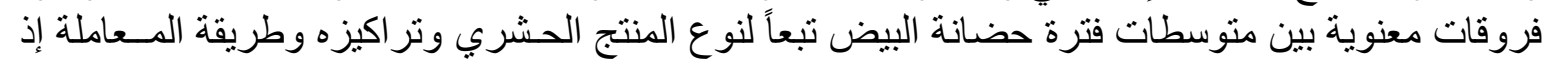


Mesopotamia. J. of Agric.

Vol.(41) No .(2) 2012
ISS:2243-4796(Online)

ISS: 1815 - 316X (Print)

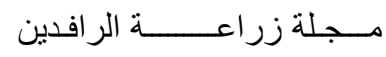

المجلد (41) العدد (2) 2012

بلغ أقصر متوسط لفترة حضانة البيض 2.61 و 2.88 يوماً للبيض الموضوع من إناث الدعاسيق المتغذية

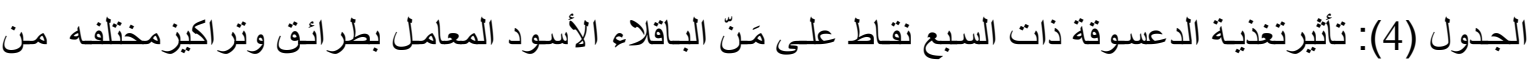

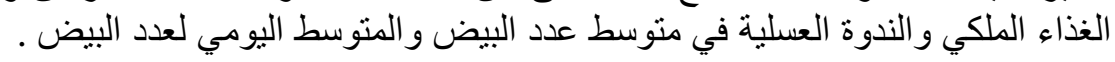

Table(4): Effect of feeding of C. septempunctata L. on Aphis fabae treated with different mehod \& different conc.of royal jelly and honeydew on mean number of eggs.

\begin{tabular}{|c|c|c|c|c|c|c|c|c|c|}
\hline \multirow{3}{*}{$\begin{array}{c}\text { التأثنير المنتج العام } \\
\text { Mean الحشرئ } \\
\text { of } \\
\text { insect product } \\
\text { effect }\end{array}$} & \multirow{3}{*}{$\begin{array}{l}\text { التر اكيز سط العام لتأثيز } \\
\text { Mean of } \\
\text { Conc. }\end{array}$} & \multicolumn{2}{|c|}{$\begin{array}{l}\text { البيض/أنثى سط اليومي لعدد } \\
\text { daily mean } \\
\text { number of egg/ femal }\end{array}$} & \multirow{3}{*}{$\begin{array}{c}\text { العام لتأثير الحشر المنتج } \\
\text { الحنتير } \\
\text { Mean of } \\
\text { insect } \\
\text { product } \\
\text { effect }\end{array}$} & \multirow{3}{*}{$\begin{array}{c}\text { العام لتأثير التير اكيز } \\
\text { Mean of } \\
\text { Conc. }\end{array}$} & \multicolumn{2}{|c|}{$\begin{array}{c}\text { متوسط عدد البيض/أنثى } \\
\text { mean number of egg/femal }\end{array}$} & \multirow{3}{*}{$\begin{array}{l}\text { Conc.\% التريز } \\
\text { Conco }\end{array}$} & \multirow{3}{*}{$\begin{array}{c}\text { نوع المنتجي } \\
\text { Kind of } \\
\text { insect } \\
\text { product }\end{array}$} \\
\hline & & $\begin{array}{c}\text { المعاملة غيرة } \\
\text { المباشرة } \\
\text { Indirect } \\
\text { Treatment }\end{array}$ & $\begin{array}{c}\text { المعاملة } \\
\text { Direct } \\
\text { Treatment }\end{array}$ & & & $\begin{array}{c}\text { المعاملة غياشرة } \\
\text { Indirect } \\
\text { Treatment }\end{array}$ & $\begin{array}{c}\text { المباشرة المعاملة } \\
\text { Direct } \\
\text { Treatment }\end{array}$ & & \\
\hline & & المتوسط & المتوسط & & & المتوسط Mean & Mean المتوسط & & \\
\hline \multirow{3}{*}{${ }^{*} 25.07 \mathrm{a}$} & $25.39 \mathrm{a}$ & $25.46 \mathrm{ab}$ & $25.31 \mathrm{a}-\mathrm{c}$ & \multirow{3}{*}{$660.67 \mathrm{a}$} & $647 b c$ & $641.20 \mathrm{~cd}$ & $652.80 \mathrm{~b}-\mathrm{d}$ & 2 & \multirow{3}{*}{$\begin{array}{l}\text { الغذاء } \\
\text { Royal } \\
\text { jelly }\end{array}$} \\
\hline & $25.16 \mathrm{ab}$ & $25.09 a-c$ & $25.22 \mathrm{a}-\mathrm{c}$ & & $661.50 \mathrm{ab}$ & $652.20 \mathrm{~b}-\mathrm{d}$ & $670.80 \mathrm{ab}$ & 4 & \\
\hline & $24.67 \mathrm{c}$ & $24.47 \mathrm{~d}$ & $24.87 b-d$ & & $673.50 \mathrm{a}$ & $665.60 \mathrm{a}-\mathrm{c}$ & $681.40 \mathrm{a}$ & 8 & \\
\hline \multirow{3}{*}{$25.11 \mathrm{a}$} & $25.34 \mathrm{ab}$ & $25.16 a-c$ & $25.51 \mathrm{a}$ & \multirow{3}{*}{$647.40 \mathrm{~b}$} & $633.20 \mathrm{c}$ & $628.80 \mathrm{~d}$ & $637.60 \mathrm{~d}$ & 2 & \multirow{3}{*}{\begin{tabular}{|c} 
العلدوة \\
Honeyde \\
w
\end{tabular}} \\
\hline & $25.02 \mathrm{a}-\mathrm{c}$ & $24.87 \mathrm{~b}-\mathrm{d}$ & $25.16 a-c$ & & $647.80 \mathrm{bc}$ & $641.40 \mathrm{~cd}$ & $654.20 \mathrm{~b}-\mathrm{d}$ & 4 & \\
\hline & $24.96 b c$ & $25.17 \mathrm{a}-\mathrm{c}$ & $24.74 \mathrm{~cd}$ & & $661.20 \mathrm{ab}$ & $654.40 \mathrm{~b}-\mathrm{d}$ & $668.00 \mathrm{ab}$ & 8 & \\
\hline \multirow[t]{2}{*}{$25.12 \mathrm{a}$} & $25.12 \mathrm{ab}$ & $25.12 \mathrm{a}-\mathrm{c}$ & $25.12 \mathrm{a}-\mathrm{c}$ & $602.40 \mathrm{c}$ & $602.40 d$ & $602.40 \mathrm{e}$ & $602.40 \mathrm{e}$ & $\begin{array}{c}\text { المقارنة } \\
\text { Control }\end{array}$ & \\
\hline & & $25.05 \mathrm{a}$ & $25.14 \mathrm{a}$ & & & $640.86 \mathrm{~b}$ & $652.46 \mathrm{a}$ & $\begin{array}{l}\text { التأثتير طريقة العامة } \\
\text { المعاملة } \\
\text { Mean of } \\
\text { Treatment } \\
\text { method }\end{array}$ & \\
\hline
\end{tabular}

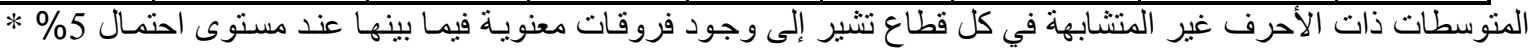
حسب اختبار دنكن.

*Means followed by different letters in each sector are significantly different at $\mathrm{P}=0.05$

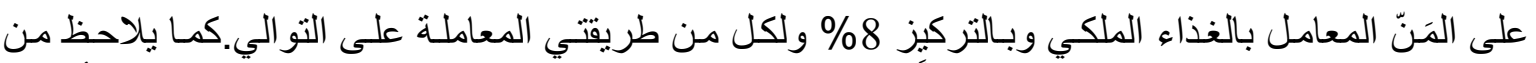

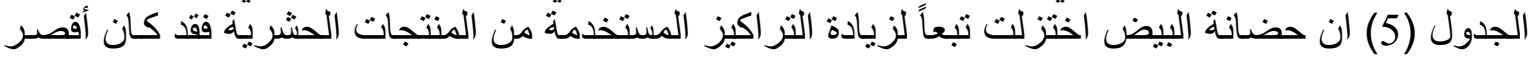

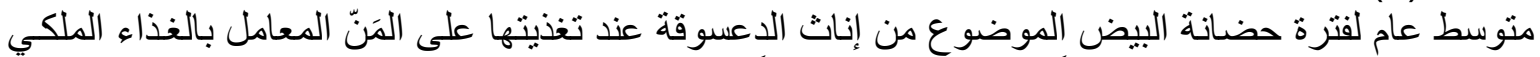

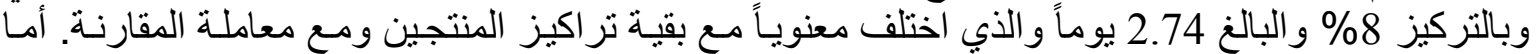

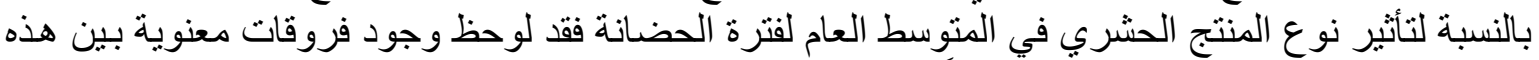

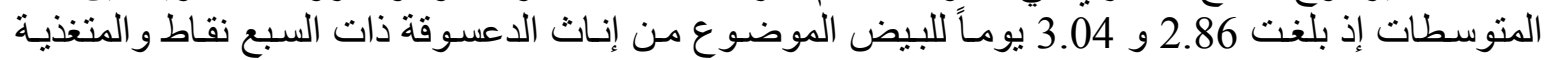

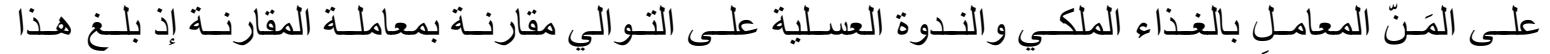

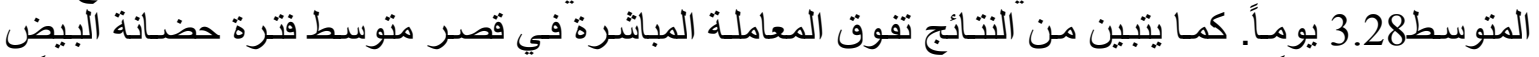

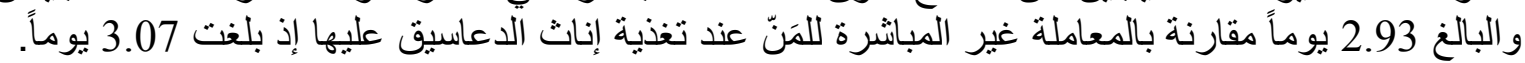

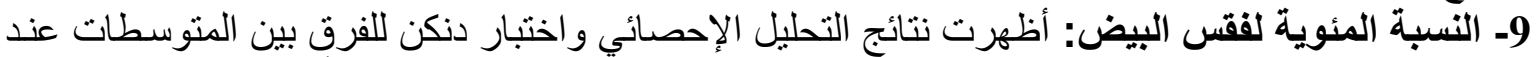

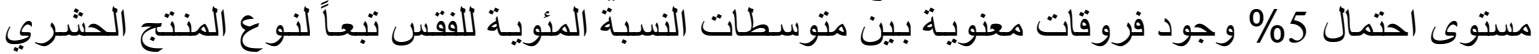

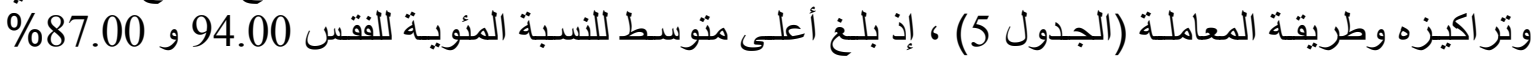

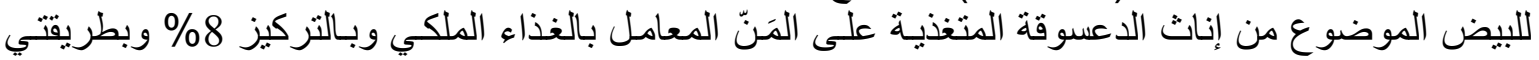


Mesopotamia. J. of Agric.

Vol.(41) No .(2) 2012
ISS:2243-4796(Online)

ISS: $1815-316 \mathrm{X}$ (Print)

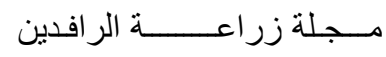

المجلد (41) العدد (2) 2012

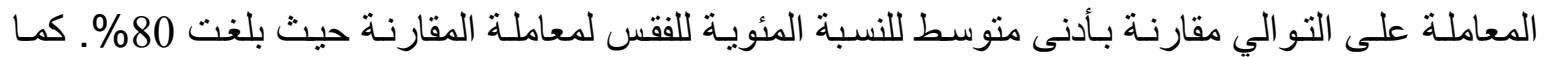

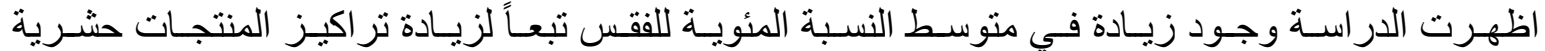

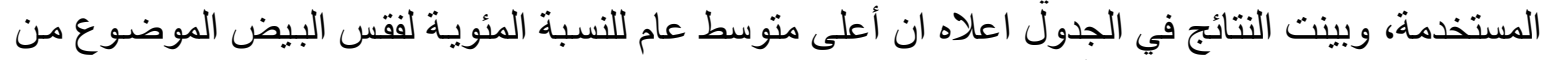

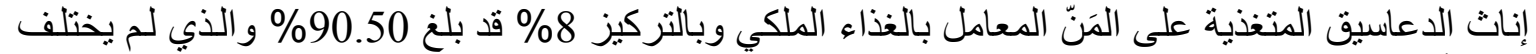

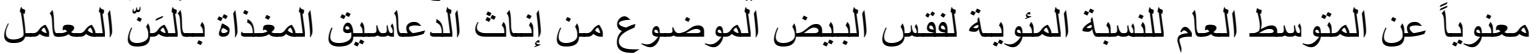

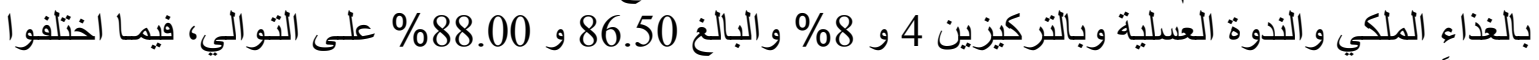

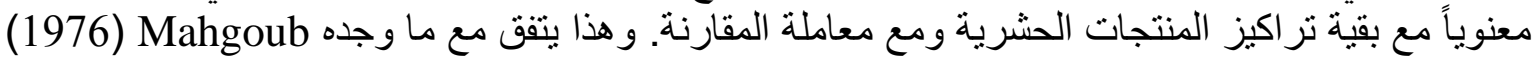
بوجود زيادة في متوسط النسبة المئوية لفقس بيض دودة حرير الخروع ع. A. ricini Boisd بزيادة التر اكيز

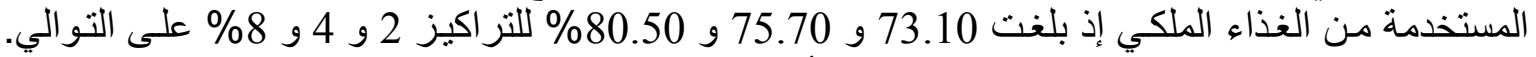
وكذلك مع ما وجده Tang وآخرون (2005) من أن تغذية برقات

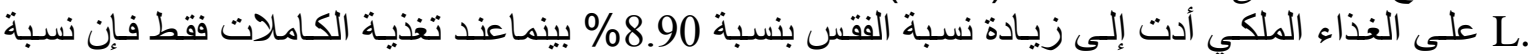

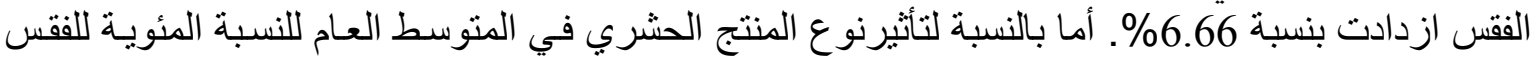

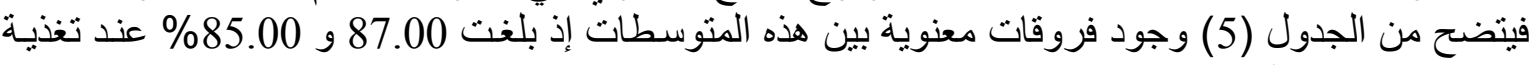

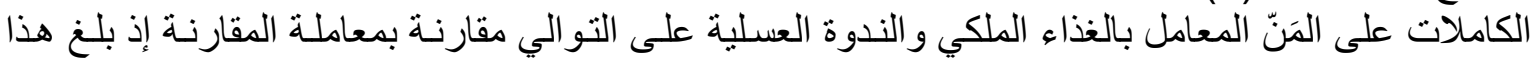

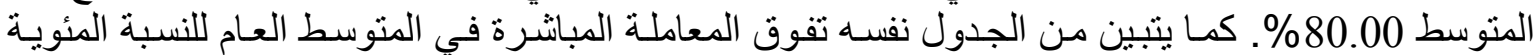

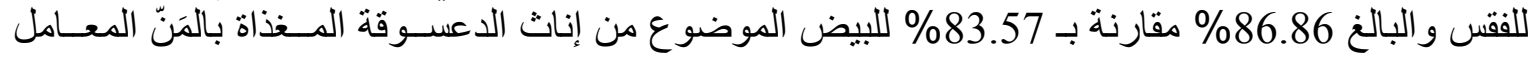

الجدول(5): تأثثرتغذية الدعسوقة ذات السبع نقاط على مَنّ الباقلاء الأسود المعامل بطر ائق وتر اكيزمختلفه

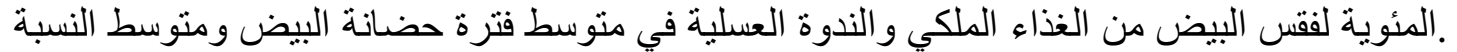

Table (5): Effect of feeding of C. septempunctata L. on Aphis fabae treated with different mehod \& different conc.of royal jelly and honeydew on means of eggs incubation period and hatching percentage.

\begin{tabular}{|c|c|c|c|c|c|c|c|c|c|}
\hline \multirow{3}{*}{$\begin{array}{c}\text { النتأثمام } \\
\text { المنتير } \\
\text { الحشري } \\
\text { Mean } \\
\text { of } \\
\text { insect } \\
\text { product }\end{array}$} & \multirow{3}{*}{$\begin{array}{c}\text { التنألتام التزير } \\
\text { التيز } \\
\text { Mean } \\
\text { of } \\
\text { Conc. }\end{array}$} & \multicolumn{2}{|c|}{$\begin{array}{c}\text { متوسط النسبة المئوية لفقس \% } \\
\text { \% البيض } \\
\text { hatching percentage\% }\end{array}$} & \multirow{3}{*}{$\begin{array}{c}\text { العام لتأثير } \\
\text { الحنتج } \\
\text { الحشري } \\
\text { Mean } \\
\text { of } \\
\text { insect } \\
\text { product }\end{array}$} & \multirow{3}{*}{ 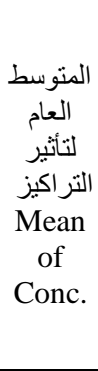 } & \multicolumn{2}{|c|}{$\begin{array}{l}\text { متوسط فترة حضانة البيض بالإم } \\
\text { incubation period/day }\end{array}$} & \multirow{3}{*}{$\begin{array}{l}\text { \% التر اكيز \% } \\
\text { Conc. }\end{array}$} & \multirow{3}{*}{$\begin{array}{l}\text { نوع المنتج الحشري } \\
\text { Kind of insect } \\
\text { product }\end{array}$} \\
\hline & & $\begin{array}{c}\text { المعاملة غيرة } \\
\text { Indirect } \\
\text { Treatment }\end{array}$ & $\begin{array}{c}\text { المعاملة } \\
\text { Direct } \\
\text { Treatment }\end{array}$ & & & \begin{tabular}{|c|} 
المعاملة غيرة \\
المبرة \\
Indirect \\
Treatment
\end{tabular} & $\begin{array}{c}\text { المباشرة } \\
\text { Direct } \\
\text { Treatment }\end{array}$ & & \\
\hline & & المتوسط Mean & $\begin{array}{l}\text { المتوسط } \\
\text { Mean }\end{array}$ & & & $\begin{array}{l}\text { المتوسط } \\
\text { Mean }\end{array}$ & $\begin{array}{l}\text { المنوسط } \\
\text { Mean }\end{array}$ & & \\
\hline \multirow{3}{*}{ *87.00a } & $\begin{array}{c}84.00 \\
\text { b-d }\end{array}$ & $83.00 \mathrm{~cd}$ & $85.00 \mathrm{~b}-\mathrm{d}$ & \multirow{3}{*}{$2.86 a$} & $2.96 \mathrm{~cd}$ & $3.06 \mathrm{e}-\mathrm{g}$ & $2.86 b c$ & 2 & \multirow{3}{*}{$\begin{array}{c}\text { الغذاء الملكي } \text { Royally } \\
\text { Rollo }\end{array}$} \\
\hline & $\begin{array}{c}86.50 \\
a-c\end{array}$ & $85.00 \mathrm{~b}-\mathrm{d}$ & $88.00 \mathrm{a}-\mathrm{c}$ & & $2.87 \mathrm{~b}$ & $2.95 \mathrm{~cd}$ & $2.79 \mathrm{~b}$ & 4 & \\
\hline & $90.50 \mathrm{a}$ & $87.00 \mathrm{~b}-\mathrm{d}$ & $94.00 \mathrm{a}$ & & $2.74 a$ & $2.88 \mathrm{bc}$ & $2.61 \mathrm{a}$ & 8 & \\
\hline \multirow{3}{*}{$85.00 \mathrm{~b}$} & $82.50 \mathrm{~cd}$ & $82.00 \mathrm{~cd}$ & $83.00 \mathrm{~cd}$ & \multirow{3}{*}{$3.04 b$} & $3.16 \mathrm{e}$ & $3.22 \mathrm{~h}$ & $3.10 \mathrm{~g}$ & 2 & \multirow{3}{*}{ الندوة العسلية } \\
\hline & $85.00 \mathrm{bc}$ & $83.00 \mathrm{~cd}$ & $87.00 \mathrm{~b}-\mathrm{d}$ & & $3.03 \mathrm{~d}$ & $3.09 \mathrm{fg}$ & $2.97 \mathrm{c}-\mathrm{e}$ & 4 & \\
\hline & $88.00 \mathrm{~b}$ & $85.00 \mathrm{~b}-\mathrm{d}$ & $91.00 \mathrm{ab}$ & & $2.93 \mathrm{bc}$ & $2.99 \mathrm{~d}-\mathrm{f}$ & $2.87 \mathrm{bc}$ & 8 & \\
\hline \multirow[t]{2}{*}{$80.00 \mathrm{~d}$} & $80.00 \mathrm{~d}$ & $80.00 \mathrm{~d}$ & $80.00 \mathrm{~d}$ & $3.28 \mathrm{c}$ & $3.28 \mathrm{f}$ & $3.28 \mathrm{~h}$ & $3.28 \mathrm{~h}$ & \multicolumn{2}{|c|}{ المقارنةControl } \\
\hline & & $83.57 \mathrm{~b}$ & $86.86 \mathrm{a}$ & & & $3.07 \mathrm{~b}$ & $2.93 a$ & \multicolumn{2}{|c|}{$\begin{array}{c}\text { المتوسط العام لتأثير طريقة المعاملة } \\
\text { Mean of Treatment } \\
\text { Method }\end{array}$} \\
\hline
\end{tabular}

*المتوسطات ذات الأحرف غير المتثابهة في كل قطاع تثير إلى وجود فروقات معنوية فيما بينها عند مستوى احتمال 5\%

Means followed by different letters in each sector are significantly different at $\mathrm{P}=0.05$

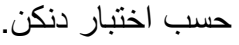




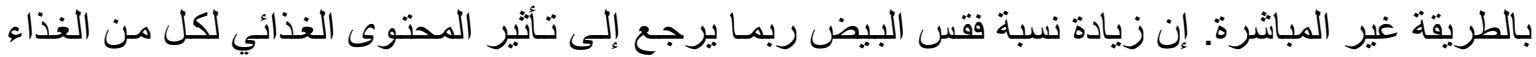

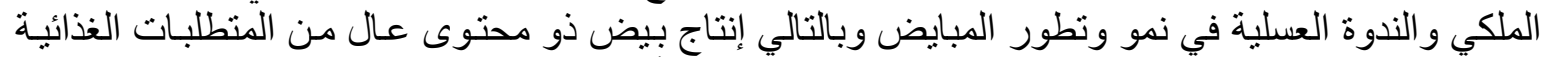

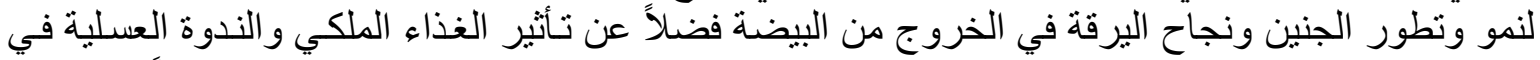

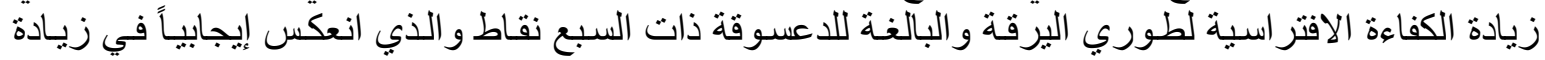

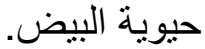

\title{
EFFECT OF THE FEEDING OF Coccinella septempunctata L. ON Aphis fabae Scopoli TREATED WITH DIFFERENT CONCENTRATION AND METHODS OF ROYAL JELLY AND HONEYDEW ON ITS SOME BIOLOGICAL CHARACTERS
}

Nazar M. AL-Mallah Juma'a T. Mohammad

Plant prot. Dept., College of Agric. and Forestry, Mosul Univ., Mosul, Iraq E-mail: naz53ar_almlaah@yahoo.com

\begin{abstract}
The feeding of the Lady beetle Coccinella septempunctata L. on black bean aphid Aphis fabae Scolopi which was treated directly and indirectly by three concentration $(2,4,8 \%)$ of royal jelly of the bee and honeydew which excreted by the aphid Pterochlorus persicae (Chold.). The results of this study showed that the ladybeetle fed on black bean aphid treated directly with royal jelly of $8 \%$ conc. produced a significant effects on the means of larval development period and pupal period which was $2.10,2.50,2.50,3.30$ and 3.80 days for 1st, 2nd, 3rd, 4th Instars and pupal stage period respectively, in comparison with 2.90, 2.90, 2.90, 4.00 and 4.30 days respectively for control treatment and showed a significant effects on the means of pre - oviposition period, oviposition period, number of egg per female, incubation period and eggs hatching percentage which reached 4.60, 27.40, 681.40, 2.61 and $94.00 \%$ respectively, compared with $6.60,24.00,602.40,3.28$, and $80 \%$ respectively for the control.

Key words: Royal jelly, Honeydew, Biological studies, Aphis fabae, Coccinella septempunctata.
\end{abstract}

Received : 24/10/2011 Accepted: 9/4/2012.

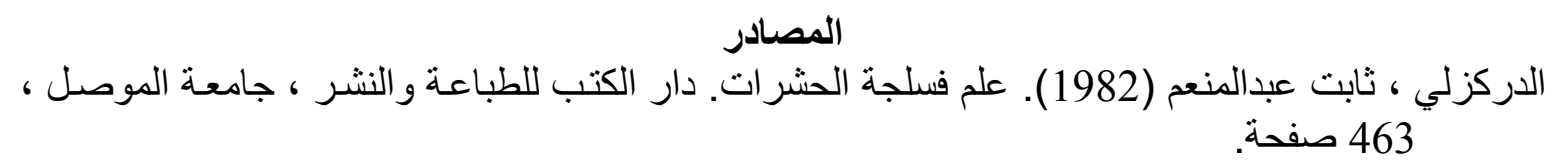

Abdul - Satar, S., N. A., Al-Saadi, A., Quda, H. S., Al-Haidari and A., Al-Azzawi (1988). Insect predators of Aphis nerii in Baghdad area and their predatory efficiency and feeding preference. Journal Of Biological Science Research. 19: $31-40$.

Anonymous (1993). SAS User's Guide : Statistics SAS Institute Inc., Cary, North Carolina, Pages 1025, USA.

Frieier, B., M. Möwes \& H. Triltsch (1998). Beneficial thresholds for Coccinella 7punctata L. (Coleoptera : Coccinellidae) as a predator of cereal aphids in winter wheat-results of population investigation and computer simulation. Journal Of Applied Entomology. 122 : 213-217. 
Hodek, I. (1967). Bionomic and ecology of predaceous Coccinellidae. Annual Review Entomology. 12 : 79-104.

Hodek, I. and A. Honĕk (1996). Ecology Of Coccinellidae. Kluwer Academic Publisher Dordrecht Boston London. 464 PP. .

Kalushkov, P. and I. Hodek. (2004). The effects of thirteen species of aphids on some life history parameters of the Ladybrid Coccinella septempunctata. Biocontrol. $49(1): 21-32$.

Kheir, A.M. (1968). Biological Studies On The Effect Of Royal Jelly. Msc. Thesis, Faculty Agriculture, Cairo University. PP. 170.

Mahgoub, M.S. (1976) : Studies On Royal Jelly. PhD. Thesis. College of Agriculture, University of Alexandria. 139 PP.

McEwen, P. K., M. A. Jervis and N. A. C. Kidd. (1993). Influence of artificial honeydew on larval development and survival in Chysoperla carnea (Neuroptera : Chrysopidae). Entomophaga. 38: 241 - 244.

Okada, I.S. and K. Minawa (1965). The effect of royal jelly on the Drosophila. Bulletin Faculty Of Agriculture Tamagawa University. (6) : 31.

Omkar, G.M. and A. Pervez (2002). Ecology of aphidophagous ladybird beetle, Coccinella septempunctata L. (Coleoptera: Coccinellidae): A review. Journal Of Aphidology. $16: 175-201$.

Singh, T.V.K., K.M. Sigh, and R.N. Singh. (1991). Influence of intercropping : III Natural enemy complex in groundnut. Indian Journal Of Entomology. 53 : $333-368$.

Triltsch, H. (1997). The ladybird Coccinella septempunctata L. within the winder wheat/cereal aphid/antagonist complex. Agrarökologie. 24, 159.

Wang L., D. Juan, Z. ShuJie, G. Jin and S. WenJie (2005). Effect of carrot and royal jelly on fecundity of Tenebrio molitor. Chinese Bulletin Of Entomology. 42 (4) : $434-438$. 